\title{
On the interplay between graphs and matroids
}

\author{
James Oxley
}

\begin{abstract}
"If a theorem about graphs can be expressed in terms of edges and circuits only it probably exemplifies a more general theorem about matroids." This assertion, made by Tutte more than twenty years ago, will be the theme of this paper. In particular, a number of examples will be given of the two-way interaction between graph theory and matroid theory that enriches both subjects.
\end{abstract}

\section{Introduction}

This paper aims to be accessible to those with no previous experience of matroids; only some basic familiarity with graph theory and linear algebra will be assumed. In particular, the next section introduces matroids by showing how such objects arise from graphs. It then presents a minimal amount of theory to make the rest of the paper comprehensible. Throughout, the emphasis is on the links between graphs and matroids.

Section 3 begins by showing how 2-connectedness for graphs extends naturally to matroids. It then indicates how the number of edges in a 2-connected loopless graph can be bounded in terms of the circumference and the size of a largest bond. The main result of the section extends this graph result to matroids. The results in this section provide an excellent example of the two-way interaction between graph theory and matroid theory.

In order to increase the accessibility of this paper, the matroid technicalities have been kept to a minimum. Most of those that do arise have been separated from the rest of the paper and appear in two separate sections, 4 and 10, which deal primarily with proofs. The first of these sections outlines the proofs of the main results from Section 3.

Section 5 begins a new topic, that of removing a cycle from a graph while maintaining the connectivity. The seed for the results in this section is a 1974 graph theorem of Mader. Various extensions and non-extensions of this theorem for 2-connected graphs and matroids are described. The topic of removable cycles continues in Section 6 with the focus moving to the 3-connected case. Once again, the symbiosis between graph theory and matroid theory should be apparent throughout this discussion.

Sections 7-10 turn attention to graph minors and their matroid analogues. In particular, motivated by Robertson and Seymour's Graph-Minors Project and a longstanding matroid conjecture of Rota, the theme is the existence of infinite antichains in graphs and matroids. The ideas of branch-width for graphs and matroids are introduced and the effects are considered both of 
bounding the branch-width above and of bounding it below. The most technical material in this discussion appears in Section 10 where some proofs are outlined. Finally, Section 11 revisits the topic of unavoidable structures, which were considered in the 2-connected case in Section 3. In particular, the substructures that are guaranteed to appear in all sufficiently large 3-connected graphs and matroids are specified.

\section{The bare facts about matroids}

This section gives a basic introduction to matroid theory beginning with a description of how matroids arise from graphs. The reader who is familiar with matroids may wish to go directly to Section 3. A more detailed treatment of the material in this section may be found in [39], the terminology and notation of which will be followed here.

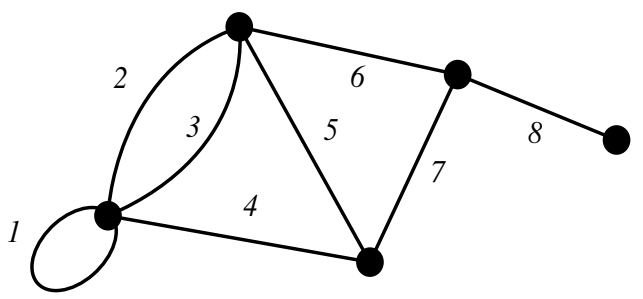

Figure 1.

Consider the graph $G$ shown in Figure 1 and let $\mathcal{C}$ be the collection of edge-sets of cycles, simple closed curves, in $G$. Then

$$
\mathcal{C}=\{\{1\},\{2,3\},\{2,4,5\},\{3,4,5\},\{5,6,7\},\{2,4,6,7\},\{3,4,6,7\}\} .
$$

Let $E$ be the edge-set of $G$. Then the pair $(E, \mathcal{C})$ is an example of a matroid. It is called the cycle matroid of $G$ and is denoted by $M(G)$.

Another example of a matroid arises from a matrix. Consider the following matrix $A$ over $G F(2)$, the field of two elements.

\begin{tabular}{|c|c|c|c|c|c|c|c|}
\hline & 1 & 2 & 3 & 4 & 5 & b & 7 \\
\hline$l$ & 0 & 1 & 1 & 1 & 0 & 0 & 0 \\
\hline & 0 & 1 & 1 & O & 1 & 1 & 0 \\
\hline & 0 & 0 & 0 & 1 & 1 & 0 & 1 \\
\hline & 0 & 0 & 0 & 0 & 0 & 1 & 1 \\
\hline & 0 & 0 & 0 & 0 & 0 & 0 & 0 \\
\hline
\end{tabular}

Let $E=\{1,2, \ldots, 8\}$ and let $\mathcal{C}$ be the collection of minimal linearly dependent subsets of $E$. Again, $(E, \mathcal{C})$ is a matroid. This matroid is called the vector matroid $M[A]$ of the matrix $A$. This matrix is the mod 2 vertex-edge incidence matrix of the graph $G$ in Figure 1, and its vector matroid equals the cycle 
matroid of $G$. Because this matrix $A$ is over the field $G F(2)$, the associated vector matroid is an example of a binary matroid.

In general, a matroid consists of a finite set $E$, called the ground set, and a collection $\mathcal{C}$ of non-empty incomparable subsets of $E$, called circuits, that obey the straightforward elimination axiom: if $C_{1}$ and $C_{2}$ are distinct circuits and $e \in C_{1} \cap C_{2}$, then $\left(C_{1} \cup C_{2}\right)-\{e\}$ contains a circuit.

The reader will easily verify that this axiom is satisfied by the collection of edge-sets of cycles in a graph and by the collection of minimal linearly dependent sets of columns of a matrix. Indeed, when Whitney [61] introduced matroids in 1935, he sought to provide a unifying abstract treatment of dependence in graph theory and linear algebra. Thus, since their introduction, matroids have been closely tied to graphs. This paper will explore some aspects of this bond.

Another basic example of a matroid is the uniform matroid $U_{r, n}$ where $r$ and $n$ are non-negative integers and $r \leq n$. This matroid has ground set $E=\{1,2, \ldots, n\}$ and has $\mathcal{C}$ equal to the set of $(r+1)$-element subsets of $E$.

While the cycle matroid of a graph is most naturally described in terms of its circuits, it is perhaps more natural to describe the vector matroid of a matrix in terms of its independent sets. These are the subsets of the ground set that contain no circuit. In the case of the vector matroid of a matrix, a set of column labels is independent if and only if the corresponding set of columns is linearly independent. A matroid is also determined by its bases, that is, its maximal independent sets. For the cycle matroid of a connected graph $G$, the bases are precisely the edge-sets of the spanning trees of $G$. Thus all the bases of $M(G)$ have the same cardinality, namely $|V(G)|-1$. This observation generalizes to arbitrary matroids: all bases of a matroid $M$ have the same cardinality, which is called the $\operatorname{rank} r(M)$ of $M$. A set $X$ spans $M$ if $X$ contains a basis of $M$.

One of the fundamental operations in graph theory is the construction of a planar dual of a plane graph. This is illustrated in Figure 2 for the graph $G$ in Figure 1. The cycle matroid $M\left(G^{*}\right)$ of $G^{*}$ has the same ground set $E$ as $M(G)$. Its circuits, the edge-sets of cycles of $G^{*}$, are $\{8\},\{6,7\},\{4,5,6\},\{4,5,7\}$, $\{2,3,4\},\{2,3,5,6\}$ and $\{2,3,5,7\}$. In the original graph $G$, these sets correspond to minimal edge-cuts or bonds, that is, they are the minimal sets of edges of $G$ whose removal increases the number of connected components of the graph. Since the graph $G$ is connected, its bonds are the minimal sets of edges whose removal disconnects the graph. Because the set $\mathcal{C}^{*}$ of bonds of $G$ equals the set of cycles of $G^{*}$, the pair $\left(E, \mathcal{C}^{*}\right)$ is a matroid. In general, for a graph $G$, planar or not, the set of bonds of $G$ is the set of circuits of a matroid on $E$, the edge-set of $G$. This matroid is called the dual matroid of $M(G)$ and is denoted by $M^{*}(G)$. 
James Oxley

(a)

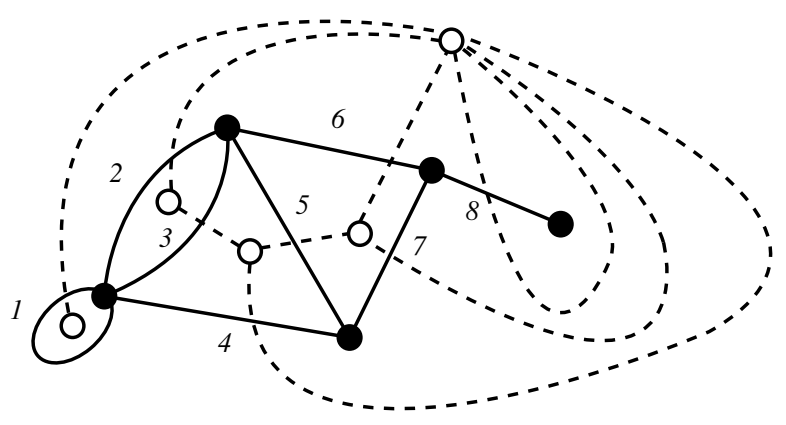

(b)

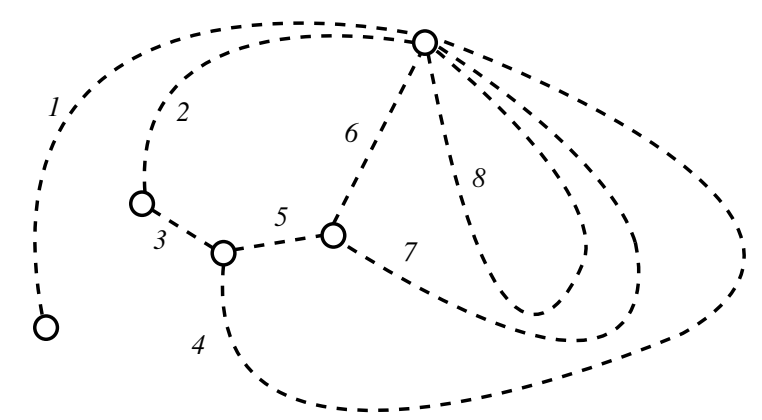

Figure 2. (a) $G$ and $G^{*}$ superimposed (b) $G^{*}$

Every matroid $M$ has a dual $M^{*}$, which has the same ground set $E$ as $M$ and has, as its bases, the collection of complements of bases of $M$. Thus the rank of $M^{*}$, which is called the corank of $M$ and is denoted by $r^{*}(M)$, equals $|E|-r(M)$. It is clear that

$$
\left(M^{*}\right)^{*}=M \text {. }
$$

The circuits of $M^{*}$ are called the cocircuits of $M$. The cocircuits of $M$ are precisely the complements of the hyperplanes, the latter being the maximal subsets of $E(M)$ that do not span $M$. For a graph $G$, the cocircuits of $M(G)$ are the bonds of $G$. It is not difficult to see that a cycle and a bond in a graph cannot have exactly one common edge. This property extends to matroids and is often referred to as orthogonality: a circuit and a cocircuit of a matroid cannot have exactly one common element. It follows from the definition that the dual of every uniform matroid is also uniform. In particular,

$$
U_{r, n}^{*}=U_{n-r, n} .
$$

The cocircuits of $U_{r, n}$ are all $(n-r+1)$-element subsets of the ground set.

Certain operations on a matrix do not alter its vector matroid. These include elementary row operations, column permutations, and deletion of a zero row. If $M$ is the vector matroid of an $r \times n$ matrix $\left[I_{r} \mid D\right]$ over a field $\mathbb{F}$, then $M^{*}$ is the vector matroid of the matrix $\left[-D^{T} \mid I_{n-r}\right]$, where the columns of this matrix are labelled in the same order as those of $\left[I_{r} \mid D\right]$.

The following table summarizes the four different classes of matroids introduced above. 


\begin{tabular}{|l|l|l|l|}
\hline Name & Notation & Ground set $E$ & Set $\mathcal{C}$ of circuits \\
\hline $\begin{array}{l}\text { cycle matroid } \\
\text { of graph } G\end{array}$ & $M(G)$ & edge-set of $G$ & $\begin{array}{l}\text { edge-sets of } \\
\text { cycles of } G\end{array}$ \\
\hline $\begin{array}{l}\text { bond matroid } \\
\text { of graph } G\end{array}$ & $M^{*}(G)$ & edge-set of $G$ & bonds of $G$ \\
\hline $\begin{array}{l}\text { vector matroid } \\
\text { of matrix } A\end{array}$ & $M[A]$ & $\begin{array}{l}\text { column labels } \\
\text { of } A\end{array}$ & $\begin{array}{l}\text { minimal linearly } \\
\text { dependent sets of columns }\end{array}$ \\
\hline uniform matroid & $U_{r, n}$ & $\{1,2, \ldots, n\}$ & $\begin{array}{l}\text { all }(r+1) \text {-element } \\
\text { subsets of }\{1,2, \ldots, n\}\end{array}$ \\
\hline
\end{tabular}

A matroid $M$ with ground set $E(M)$ is graphic if it is isomorphic to the cycle matroid of some graph, that is, there is a graph $G$ and a bijection $\phi$ : $E(M) \rightarrow E(G)$ such that a subset $X$ of $E(M)$ is a circuit of $M$ if and only if $\phi(X)$ is the edge set of a cycle of $G$. A cographic matroid is one that is isomorphic to the bond matroid of some graph. For a field $\mathbb{F}$, a matroid is $\mathbb{F}$-representable if it is isomorphic to the vector matroid of some matrix over $\mathbb{F}$.

Duality is one of the three most basic matroid operations. The other two, deletion and contraction, are, like the first, extensions of natural graph operations.
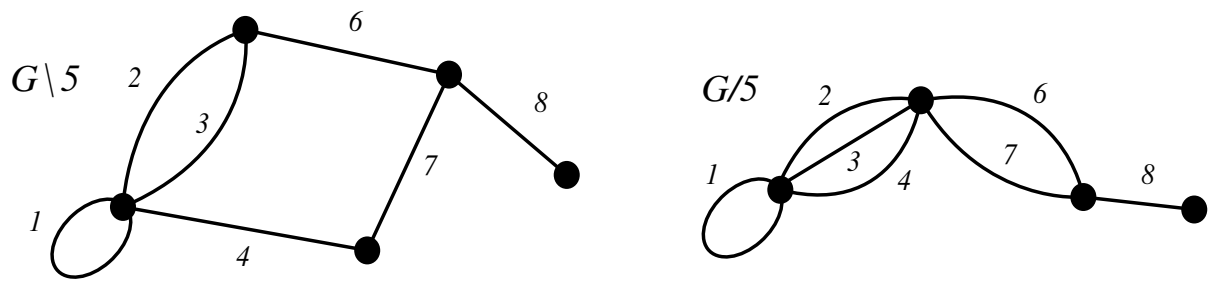

Figure 3.

The two graphs in Figure 3 are related to the graph $G$ in Figure 1. The first of these, denoted $G \backslash 5$, is obtained from $G$ by deleting the edge 5 ; the second, denoted $G / 5$, is obtained by contracting 5 , that is, by shrinking the edge 5 to a single vertex, or (equivalently) deleting 5 and then identifying its end-vertices.

To extend these definitions to matroids, we consider how the cycles of $G \backslash e$ and $G / e$ are related to those of $G$. In general, if $T$ is a subset of the ground set $E$ of a matroid $M$, then $M \backslash T$, the deletion of $T$ from $M$, is the matroid with ground set $E-T$ whose set of circuits is $\{C \in \mathcal{C}(M): C \cap T=\emptyset\}$; the contraction of $T$ from $M$, which is denoted $M / T$, also has $E-T$ as its ground set, but its circuits are the minimal non-empty members of $\{C-T: C \in$ $\mathcal{C}(M)\}$. It is straightforward to check that both $M \backslash T$ and $M / T$ are actually matroids and that if $e$ is an edge of a graph $G$, then $M(G) \backslash e=M(G \backslash e)$, while $M(G) / e=M(G / e)$. If $X$ is a subset of $E$, then all the bases of $M \backslash(E-X)$ 
have the same cardinality, which equals the rank of $M \backslash(E-X)$ and is also called the $\operatorname{rank} r(X)$ of $X$.
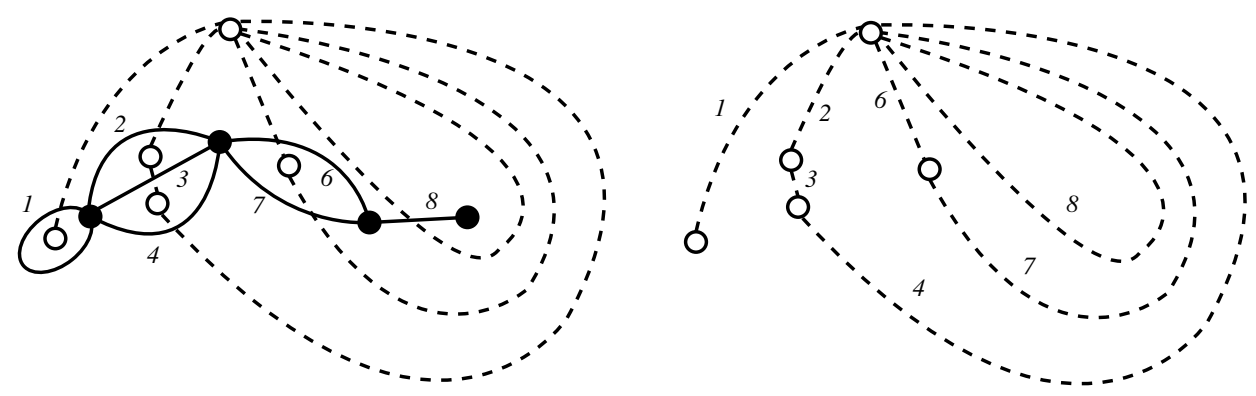

Figure 4.

For graphs, deletion and contraction are related through duality. In particular, Figure 4 shows that the graphs $(G / 5)^{*}$ and $G^{*} \backslash 5$ are equal. This relationship extends to matroids. Specifically, if $T$ is a subset of the ground set of a matroid $M$, then

$$
M^{*} \backslash T=(M / T)^{*} \text {, or (equivalently) } M \backslash T=\left(M^{*} / T\right)^{*} .
$$

A graph $H$ is a minor of a graph $G$ if $H$ can be obtained from $G$ by a sequence of deletions, contractions, and deletions of isolated vertices. There is a corresponding notion for matroids with the only difference arising because arbitrary matroids do not have vertices. Thus a minor of a matroid $M$ is any matroid that can be obtained from $M$ by a sequence of deletions and contractions. It is not difficult to check that if $X$ and $Y$ are disjoint subsets of the ground set of a matroid $M$, then

$$
M \backslash X / Y=M / Y \backslash X,
$$

and every minor of $M$ is determined by the set of elements that are deleted and the set of elements that are contracted. Although it is true that if the graph $G_{1}$ is a minor of the graph $G_{2}$, then the matroid $M\left(G_{1}\right)$ is a minor of the matroid $M\left(G_{2}\right)$, the converse of this fails. For example, if $G_{1}$ is the union of two disjoint copies of $K_{2}$ with edge sets $\{1\}$ and $\{2\}$, and $G_{2}$ is a copy of $K_{3}$ with edge set $\{1,2,3\}$, then $M\left(G_{1}\right) \cong U_{2,2}$ and $M\left(G_{2}\right) \cong U_{2,3}$. Certainly $M\left(G_{1}\right)$ is a minor of $M\left(G_{2}\right)$. However, clearly $G_{1}$ has four vertices while $G_{2}$ has three, so $G_{1}$ is not a minor of $G_{2}$.

Matroids also arise geometrically as follows. Given a finite set $E$ of points, such as the seven points of the Fano projective plane (see Figure 5(b)), one can define a matroid $M$ on $E$ by first choosing a collection of subsets of $E$ called lines such that two distinct lines share at most one common point. The circuits of $M$ are all sets of three collinear points and all sets of four points no three of which are collinear. Such a matroid has rank at most three. When $M$ is derived in this way from the Fano plane, with the lines of the matroid being 
the seven lines of the projective plane, including $\{4,5,6\}$, we call $M$ the Fano matroid $F_{7}$. Another important example of such a matroid is the non-Fano matroid $F_{7}^{-}$which is represented geometrically in Figure 5(a). It is obtained from $F_{7}$ by relaxing $\{4,5,6\}$, which is both a circuit and a hyperplane of $F_{7}$. In general, if $X$ is a circuit-hyperplane in a matroid $M$, then one can obtain a new matroid $M^{\prime}$ from $M$ by relaxing $X$, that is, by declaring $X$ to be an independent set but leaving the matroid unchanged otherwise. For example, if we apply this operation six times to $F_{7}^{-}$, we obtain the uniform matroid $U_{3,7}$.
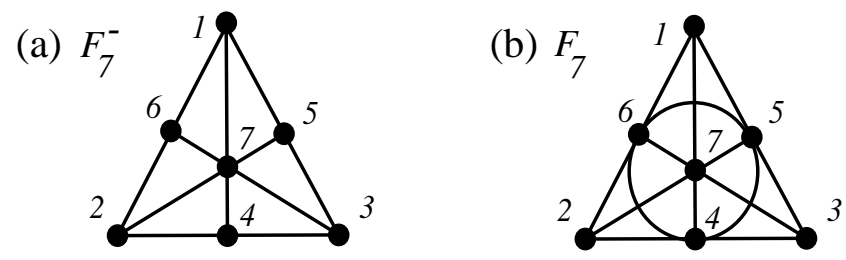

Figure 5 .

The Fano matroid is isomorphic to the vector matroid of the matrix whose columns consist of all non-zero vectors in the 3-dimensional vector space over $G F(2)$. Hence the Fano matroid is binary where, in general, a matroid $M$ is binary if $M$ is isomorphic to the vector matroid of some matrix over the field $G F(2)$. By using the mod-2 vertex-edge incidence matrix, as in the first example, it is straightforward to show that every graphic matroid is binary. The Fano matroid and its dual are examples of non-graphic binary matroids. To see that $F_{7}$ is non-graphic, it suffices to observe that there is no simple graph on 4 vertices with exactly 7 edges since the complete graph $K_{4}$ has only 6 edges. The matroid $U_{2,4}$ is non-binary because there is no $2 \times 4$ matrix $A$ over $G F(2)$ such that $U_{2,4} \cong M[A]$.

A binary matroid is regular if it has no minor isomorphic to either $F_{7}$ or $F_{7}^{*}$. Such matroids have numerous attractive properties, some of which appear in the next result. A totally unimodular matrix is a real matrix all of whose subdeterminants are in $\{0,1,-1\}$.

Proposition 2.1 The following statements are equivalent for a matroid $M$.

(i) $M$ is regular.

(ii) For all fields $\mathbb{F}$, there is a matrix $A_{\mathbb{F}}$ over $\mathbb{F}$ such that $M$ is isomorphic to the vector matroid of $A_{\mathbb{F}}$.

(iii) $M$ is isomorphic to $M[A]$ for some totally unimodular matrix $A$.

(iv) $M$ has no minor isomorphic to any of $F_{7}, F_{7}^{*}$, or $U_{2,4}$.

One very special regular matroid $R_{10}$ is the vector matroid of the matrix over $G F(2)$ whose columns are the ten distinct 5 -tuples with exactly three 
ones. In a result that underlies the proof that totally unimodular matrices can be recognized in polynomial time, Seymour [50] established that every regular matroid can be built by sticking together graphic matroids, cographic matroids, and copies of $R_{10}$ using operations that mimic sticking two graphs together at a vertex, along an edge, and across a triangle. This result means that one's best hope of obtaining some matroid extension of a graph result is to be able to extend the result to regular matroids. Some results extend further, to binary matroids, and others further still to, say, all vector matroids. Thus although a graph result may fail to extend to all matroids, there are several natural intermediate classes to which the result may extend.

\section{Connectedness, 2-connectedness, and unavoidable stuctures}

One area in which the interaction between graphs and matroids has been very fruitful is in the consideration of connectivity. Initially there is some cause for pessimism when we note that the graphs $G_{1}$ and $G_{2}$ shown in Figure 6 have the same edge sets and have the same sets of edge-sets of cycles. Thus $M\left(G_{1}\right)=M\left(G_{2}\right)$. But $G_{1}$ is a connected graph while $G_{2}$ is not. We conclude that graph connectedness has no matroid generalization. However, if we look at 2-connectedness, we note that we can describe this property for graphs in terms of edges and circuits only: a loopless graph $G$ is 2-connected if and only if, for every two edges $e$ and $f$ of $G$, there is a cycle containing $\{e, f\}$. Mimicking this result for graphs, we define a matroid to be 2-connected if, for every two elements, there is a circuit containing both. This terminology follows Tutte [56]. For many other authors, a matroid with the above property is called connected. Thus, for matroids, the terms "connected" and "2-connected" mean precisely the same thing. One very attractive feature of 2-connectedness for matroids, which is a consequence of orthogonality, is that a matroid is 2connected if and only if its dual is. In a matroid $M$, a maximal subset $X$ of $E(M)$ for which $M \backslash(E(M)-X)$ is 2-connected is called a (2-connected) component of $M$. Thus, for the graph $G_{1}$ in Figure $6, M\left(G_{1}\right)$ has exactly two components.

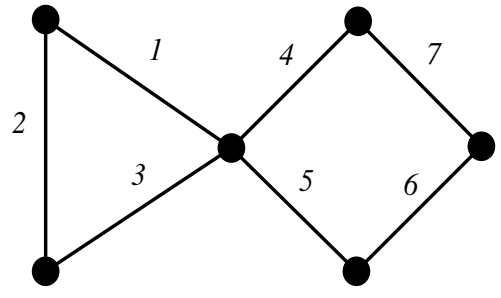

(a)

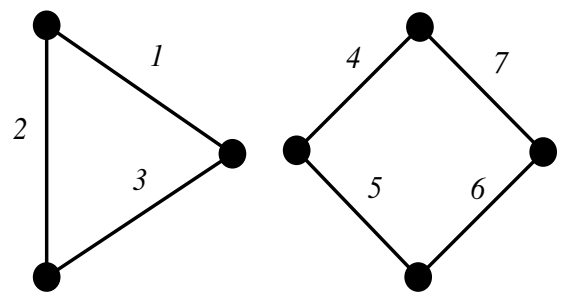

(b)

Figure 6. 
Our discussion of areas of interaction between graphs and matroids will begin with the consideration of unavoidable structures in graphs. An easy example of such a result is the observation that a big connected graph has either a long path or a vertex of high degree. More formally:

Lemma 3.1 For each positive integer $n$, there is an integer $k(n)$ such that every connected graph $G$ with at least $k(n)$ edges contains a path with at least $n$ vertices or a vertex of degree at least $n$.

As one might expect, if one imposes a stronger connectivity condition on $G$, then one can guarantee the presence of a more specific substructure. In particular, if $G$ is 2-connected, then we have the following result.

Proposition 3.2 Every sufficiently large 2-connected graph contains either a big cycle or a vertex of high degree.

In a 2-connected loopless graph $G$, the set of edges incident with a vertex forms a bond of $G$. Thus we have the following immediate consequence of the last result.

Corollary 3.3 Every sufficiently large 2-connected loopless graph contains either a big cycle or a big bond.

Our aim is to extend graph results to matroids so it is natural to ask whether the matroid analogue of the last result holds. Specifically, is it true that every sufficiently large 2-connected matroid contains either a big circuit or a big cocircuit? The question was raised informally by Robin Thomas at the Graph-Minors meeting in Seattle in 1991. It was answered very quickly by Lovász, Schrijver, and Seymour (in [39]) who proved the following result.

Theorem 3.4 Let $M$ be a 2-connected matroid with at least two elements. If a largest circuit of $M$ has c elements and a largest cocircuit has $c^{*}$ elements, then $M$ has at most $2^{c+c^{*}-1}$ elements.

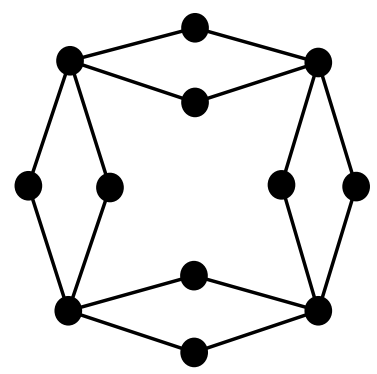

Figure 7. $G$ 
An answer to Thomas's question can also be deduced from work of Tuza [59] on set systems. A natural question that arises here is whether the bound in the last theorem can be sharpened for various special classes of matroids such as the class of graphic matroids. For example, for the cycle matroid of the graph $G$ shown in Figure $7, c$, which is just the circumference of $G$, equals 8 , while $c^{*}$ is 4 . Thus $2^{c+c^{*}-1}=2048$, although $G$ has just 16 edges.

From a matroid perspective, one advantage of the bound in Theorem 3.4 is that it is symmetric in $c$ and $c^{*}$. Duality is so fundamental in matroid theory that it is desirable to retain this property. As a guide to potential improvements in the bound for graphs, we consider what is already known for graphs. The following is an old result of Erdős and Gallai [14].

Theorem 3.5 Let $G$ be a simple n-vertex graph. Then

$$
|E(G)| \leq \frac{1}{2} c(n-1)
$$

Motivated by this result and the desire to obtain a bound symmetric in $c$ and $c^{*}$, the author asked whether, for graphs, the bound $\frac{1}{2} c c^{*}$ holds. The graph in Figure 7 shows that such a bound would be sharp. Pou-Lin Wu [62] proved this bound.

Theorem 3.6 Let $G$ be a 2-connected loopless graph with circumference $c$. If $c^{*}$ is the size of a largest bond in $G$, then

$$
|E(G)| \leq \frac{1}{2} c c^{*}
$$

The following table compares Wu's bound with the Erdös-Gallai bound.

\begin{tabular}{|l|l|l|}
\hline & Erdős-Gallai & $\mathrm{Wu}$ \\
\hline$|E(G)| \leq$ & $\frac{1}{2} c(n-1)$ & $\frac{1}{2} c c^{*}$ \\
\hline NEED & $G$ is simple & $G$ is 2-connected and loopless \\
\hline SHARP FOR & complete graphs only & cycles etc. \\
\hline
\end{tabular}

We conclude that Wu's bound, motivated by matroid theory, is often sharper than the Erdős-Gallai bound. Thus, in Theorem 3.6, we have a graph result that was motivated by a matroid result and frequently improves on the previous best graph result.

The natural question that Wu's theorem raises is whether the same bound holds for all 2-connected matroids. Wu was not able to extend his proof to binary matroids. The reader may hope that Seymour's decomposition theorem 
for regular matroids mentioned above would enable the graph result to be extended to regular matroids but this was not achieved either. Several authors $[2,4,22,23,41,62,63]$ considered this problem and, in particular, Reid [41] proved several attractive results. Eventually, Bonin, McNulty and Reid [2] conjectured that the same bound holds for all matroids. At that stage, there was not even a known bound that was polynomial in $c$ and $c^{*}$.

Such a polynomial bound can be derived from the next result of Manoel Lemos and the author [34]. If $M$ is a 2-connected matroid with at least two elements, then every element $e$ of $M$ is in a circuit and a cocircuit. Let $c_{e}$ be the size of a largest circuit containing $e$ and let $c_{e}^{*}$ be the size of a largest cocircuit containing $e$.

Theorem 3.7 Let $M$ be a 2-connected matroid with at least two elements. If $e$ is an element of $M$, then

$$
|E(M)| \leq\left(c_{e}-1\right)\left(c_{e}^{*}-1\right)+1 .
$$

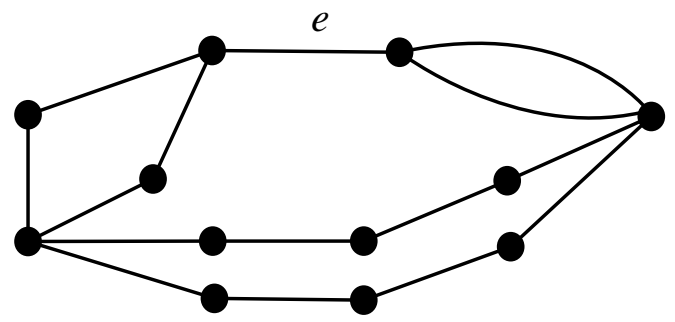

Figure 8 .

The example given in Figure 8 has $c_{e}=8$ and $c_{e}^{*}=3$. Thus $\left(c_{e}-1\right)\left(c_{e}^{*}-\right.$ $1)+1=15=|E(G)|$. Hence the bound in this theorem is sharp. All the matroids that attain this bound were determined in [34]. They are the cycle matroids of certain special series-parallel networks.

The last theorem has an attractive similarity to a bound derived from the width-length inequality $[30,16]$. In a matroid $M$, let $e$ be an element that is neither a loop, that is, a 1-element circuit, nor a coloop, a 1-element cocircuit. Let $l(M)+1$ and $w(M)+1$ be the cardinalities of a smallest circuit containing $e$ and a smallest cocircuit containing $e$. Lehman [30] showed that if $M$ is regular, then

$$
l(M) w(M)+1 \leq|E(M)| .
$$

More generally, it follows from a result of Seymour [48] that the last inequality holds for all binary matroids that have no $F_{7}^{*}$-minor using $e$. In fact, it also holds for $F_{7}^{*}$, although it fails, for example, for $A G(3,2)$. The last matroid is the matroid on the points of the three-dimensional affine space over $G F(2)$ where a set of elements is independent in the matroid if the corresponding set of points is affinely independent. Equivalently, $A G(3,2)$ is the vector matroid 
of the matrix over $G F(2)$ whose columns are all the 4-tuples whose coordinates sum to one over $G F(2)$.

A straightforward consequence of Theorem 3.7 is the following result for graphs.

Corollary 3.8 Let $u$ and $v$ be distinct vertices in a 2-connected loopless graph $G$. Then $|E(G)|$ cannot exceed the product of the length of a longest $(u, v)$-path and the size of a largest minimal edge-cut separating $u$ from $v$.

Guoli Ding, a colleague of the author at Louisiana State University, gave a short proof of Theorem 3.7 by induction. This proof was then modified by Manoel Lemos to prove the following strengthening of the theorem. The proof will be given in the next section.

Theorem 3.9 Let $M$ be a 2-connected matroid with at least two elements. If $e \in E(M)$, then $M$ has $c_{e}(M)-1$ cocircuits each containing e such that the union of these cocircuits is $E(M)$.

Using the bound Theorem 3.7 and quite a bit more work, Lemos and the author [34] proved Bonin, McNulty and Reid's conjecture. An outline of the proof will be given in the next section.

Theorem 3.10 Let $M$ be a 2-connected matroid with at least two elements. Then

$$
|E(M)| \leq \frac{1}{2} c c^{*}
$$

For comparison, a lower bound on $|E(M)|$ in terms of $c$ and $c^{*}$ that holds for all matroids $M$ having non-zero rank and corank is

$$
c+c^{*}-2 \leq|E(M)| .
$$

To see this, observe that $c \leq r(M)+1$ and $c^{*} \leq r^{*}(M)+1$. The bound in (3.2) is sharp. It is attained, for example, by all uniform matroids of non-zero rank and corank.

Neumann-Lara, Rivera-Campo, and Urrutia [37] proved the following extension of Pou-Lin Wu's result for graphs.

Theorem 3.11 Every 2-connected loopless graph $G$ with circumference c has a collection of $c$ bonds such that every edge of $G$ lies in at least two of them.

Observe that the last result allows for a bond to be repeated in the collection. It is natural to ask whether this result extends to matroids.

Question 3.12 Does every 2-connected matroid $M$ have a collection of $c(M)$ cocircuits such that every element is in at least two of them?

This question is open even for bond matroids of graphs. 
Question 3.13 Let $G$ be a 2-connected loopless graph whose largest bond has $c^{*}$ edges. Does $G$ have a collection of $c^{*}$ cycles such that every edge is in at least two of them?

Pou-Lin $\mathrm{Wu}$ [63] completely characterized all the graphs that attain equality in the bound in Theorem 3.10. They turn out to be certain special seriesparallel networks that are closely related to the graphs that attain equality in Theorem 3.7. Among arbitrary matroids, the binary affine space $A G(3,2)$ also has exactly $\frac{1}{2} c c^{*}$ elements. It is the only known example of a non-graphic matroid that attains the bound in Theorem 3.10.

\section{Some proofs}

In this section, we prove Theorem 3.9 and indicate the main steps in the proof of Theorem 3.10. Tutte [56] proved the following attractive property of 2-connected matroids that is particularly helpful in induction arguments.

Lemma 4.1 Let e be an element of a 2-connected matroid $M$. Then $M \backslash e$ or $M / e$ is 2-connected.

Proof of Theorem 3.9 We argue by induction on $c_{e}(M)$. If $c_{e}(M)=2$, then $M$ is a uniform matroid having rank one, so $E(M)$ is a cocircuit of $M$ and the result follows. Now suppose that the theorem holds for $c_{e}(M)<n$ and let $c_{e}(M)=n \geq 3$.

Let $C^{*}$ be a cocircuit of $M$ that contains $e$. Clearly $C^{*} \neq E(M)$. By Lemma 4.1, we may remove the elements of $C^{*}-e$ from $M$ one at a time by deletion or contraction so as to always maintain a 2-connected matroid. Thus, there is a partition $\{X, Y\}$ of $C^{*}-e$ such that $M \backslash X / Y$ is 2-connected. Call this minor $N$. By orthogonality, every circuit of $M$ that contains $e$ must also meet $X$ or $Y$. It follows that

$$
c_{e}(N)<c_{e}(M)
$$

Since $C^{*} \neq E(M)$, the matroid $N$ has at least two elements. Thus, by the induction assumption, for some $k \leq c_{e}(N)-1$, there are $k$ cocircuits $C_{1}^{*}, C_{2}^{*}, \ldots, C_{k}^{*}$ of $N$ each containing $e$ such that the union of these cocircuits is $E(N)$. For each $C_{i}^{*}$, there is a cocircuit $D_{i}^{*}$ of $M$ such that $C_{i}^{*}=D_{i}^{*}-X$. Hence $C^{*}, D_{1}^{*}, D_{2}^{*}, \ldots, D_{k}^{*}$ are cocircuits of $M$ each containing $e$ and

$E(M)=E(N) \cup X \cup Y \subseteq C_{1}^{*} \cup C_{2}^{*} \cup \cdots \cup C_{k}^{*} \cup C^{*} \subseteq D_{1}^{*} \cup D_{2}^{*} \cup \cdots \cup D_{k}^{*} \cup C^{*}$.

Thus we have a family of $k+1$ cocircuits of $M$ that covers $E(M)$. Since

$$
k+1 \leq\left(c_{e}(N)-1\right)+1=c_{e}(N) \leq c_{e}(M)-1,
$$


the result follows by induction.

There are two main steps in the proof of Theorem 3.10. These are stated in the next two lemmas. For a circuit $C$ in a matroid $M$ with $|C| \geq 2$, let $c^{*}(C, M)$ be the size of a largest cocircuit of $M$ that has exactly two elements in common with $C$. The components of a matroid $N$ are the maximal subsets $X$ of $E(N)$ such that $N \backslash(E(N)-X)$ is 2-connected.

Lemma 4.2 Let $M$ be a 2-connected matroid with at least two elements and let $C$ be a circuit of $M$. If every component of $M / C$ is a circuit, then

$$
|E(M)| \leq|C|+c(M)\left\lceil\frac{c^{*}(C, M)-2}{2}\right\rceil .
$$

Lemma 4.3 Let $M$ be a 2-connected matroid with at least two elements and let $C$ be a circuit of $M$. If $C=c(M)$, then

$$
|E(M)| \leq c(M)\left\lceil\frac{c^{*}(C, M)}{2}\right\rceil .
$$

The proof of Lemma 4.3, which uses Theorem 3.7, is by induction on the number of components of $M / C$ that are not circuits. If this number is zero, then Lemma 4.3 follows by Lemma 4.2. Using the second of these lemmas, it is not difficult to prove Theorem 3.10 .

Proof of Theorem 3.10 We shall first prove the theorem when $c^{*}(M)$ is even. By Lemma 4.3,

$$
|E(M)| \leq c(M)\left\lceil\frac{c^{*}(C, M)}{2}\right\rceil
$$

for any circuit $C$ of $M$ such that $|C|=c(M)$. As $c^{*}(C, M) \leq c^{*}(M)$, it follows that

$$
|E(M)| \leq c(M)\left\lceil\frac{c^{*}(C, M)}{2}\right\rceil \leq c(M)\left\lceil\frac{c^{*}(M)}{2}\right\rceil=\frac{c(M) c^{*}(M)}{2},
$$

where the last equality follows since $c^{*}(M)$ is an even integer.

When $c^{*}(M)$ is odd, let $M^{\prime}$ be the matroid obtained from $M$ by inserting an element in parallel with each element of the latter. For graphs, this operation is certainly well-defined. To see that it is well-defined for matroids in general, one needs only to perform the routine check that the collection of circuits of $M^{\prime}$ obeys the circuit elimination axiom. By orthogonality, $c^{*}\left(M^{\prime}\right)=2 c^{*}(M)$. Thus $c^{*}\left(M^{\prime}\right)$ is even and hence

$$
\left|E\left(M^{\prime}\right)\right| \leq \frac{c\left(M^{\prime}\right) c^{*}\left(M^{\prime}\right)}{2} .
$$

As $\left|E\left(M^{\prime}\right)\right|=2|E(M)|$ and $c\left(M^{\prime}\right)=c(M)$, we get

$$
2|E(M)| \leq c(M) c^{*}(M)
$$

and the result follows. 


\section{Removable circuits}

Another area in which the interaction between graphs and matroids has been quite fruitful is in the consideration of removable circuits. The following graph result of Mader [35] motivated much of what has been done here.

Theorem 5.1 Let $G$ be a simple $k$-connected graph with minimum degree at least $k+2$. Then $G$ has a cycle $C$ such that the graph obtained from $G$ by deleting all the edges of $C$ is $k$-connected.

We shall consider trying to extend this result to matroids. To do this, attention will be restricted to the cases when $k=2$ and when $k=3$. The reason for doing this is that, although Tutte defined matroid $k$-connectedness for all $k \geq 2$, we only really have a good collection of tools for working with $k$ connected matroids when $k$ is 2 or 3 . First consider 2-connected matroids. As noted earlier, in a 2-connected graph without loops, the set of edges meeting at a vertex forms a bond. Thus Mader's theorem immediately implies the following result.

Corollary 5.2 Let $G$ be a simple 2-connected graph in which every bond has size at least 4 . Then $G$ has a cycle $C$ such that $G \backslash C$ is 2-connected.

Notice that, in the last result, we are deleting the edges but not the vertices of $C$. Such a cycle $C$ in a 2-connected graph is called removable. Analogously, a circuit $D$ of a 2-connected matroid is removable if $M \backslash D$ is 2-connected. The last corollary is in an ideal form for generalization to matroids.

Question 5.3 Let $M$ be a 2-connected matroid such that

(i) every circuit has size at least 3; and

(ii) every cocircuit has size at least 4 .

Does $M$ have a removable circuit?

It is not difficult to see that the answer to this question is negative. For example, the uniform matroid $U_{3,6}$ of rank 3 on 6 elements has every circuit and every cocircuit of size 4 . But every deletion of a circuit is isomorphic to $U_{2,2}$, which is not 2-connected. When a graph result like this fails to extend to matroids in general, it is common to restrict attention to binary matroids, or if it fails even for binary matroids, to restrict attention further to regular matroids. For binary matroids, Question 5.3 appeared as an unsolved problem in [39]. In the original version of [20], Goodyn, van den Heuvel and McGuinness made the following conjecture, which would follow if Question 5.3 had an affirmative answer for the bond matroids of graphs.

Conjecture 5.4 Let $G$ be a 2-connected graph such that 
(i) every bond has size at least 3; and

(ii) every cycle has size at least 4 .

Then $G$ has a bond $C^{*}$ such that $G / C^{*}$ is 2-connected.

The addition of the requirement that $G / C^{*}$ is loopless would make this conjecture equivalent to the existence of an affirmative answer to Question 5.3 for the bond matroids of graphs.

Manoel Lemos [32] gave the following counterexample to this conjecture and thereby answered Question 5.3. Begin with $K_{5,5}$ having as its two vertex classes $\{1,2,3,4,5\}$ and $\{6,7,8,9,10\}$. For every 3 -subset $\{i, j, k\}$ of $\{1,2,3,4,5\}$ and of $\{6,7,8,9,10\}$, add two new vertices $v_{i j k}$ and $w_{i j k}$ each joined to all of $i, j, k$ and nothing else.

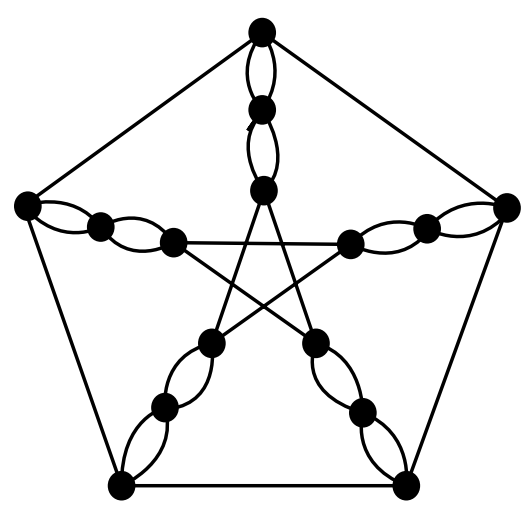

Figure 9.

Much of the impetus for the study of removable circuits in graphs seems to have been provided by a question of Arthur Hobbs as to whether every 2-connected Eulerian graph with minimum degree at least four contains a removable cycle. Robertson (in [24]) and, independently, Jackson [24] answered Hobbs's question negatively by producing the modified Petersen graph shown in Figure 9. Clearly this graph is non-simple. Jackson [24] conjectured in 1980 that it is the existence of a Petersen-graph minor that prevents the above graph from having a removable circuit.

Conjecture 5.5 Let $G$ be a 2-connected graph with minimum degree at least four. If $G$ has no minor isomorphic to the Petersen graph, then $G$ has a removable cycle.

In 1985, Fleischner and Jackson [15] proved the existence of a removable cycle in a 2-connected planar graph with minimum degree at least four. But it was not until 1997 that Jackson's conjecture was proved, by Goddyn, van den Heuvel, and McGuinness [20]. 
Graphs and matroids

Theorem 5.6 Let $G$ be a 2-connected graph with minimum degree at least four. If $G$ has no minor isomorphic to the Petersen graph, then $G$ has two edge-disjoint removable cycles.

We now return to the consideration of bonds in 2-connected graphs that can be contracted to maintain 2-connectedness. The graph in Figure 10 was identified by Goddyn, van den Heuvel, and McGuinness [20] as one having no such bond. This graph is not a counterexample to Conjecture 5.4 since it clearly has numerous bonds of size two. In response to this example, Goddyn, van den Heuvel, and McGuinness [20] conjectured the following analogue of Theorem 5.6.

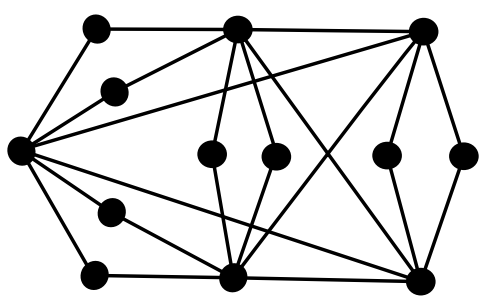

Figure 10.

Conjecture 5.7 Let $G$ be a 2-connected graph such that every cycle has at least four elements. If $G$ has no minor isomorphic to $K_{5}$, then $G$ has a bond $C^{*}$ such that $G / C^{*}$ is 2-connected.

This conjecture remains open although McGuinness [36] has proved the following partial result.

Theorem 5.8 Let $G$ be a 2-connected bipartite graph that is not a multiple edge. If $G$ has no minor isomorphic to $K_{5}$, then $G$ has a bond $C^{*}$ such that $G / C^{*}$ is 2-connected.

We know from Robertson and Jackson's example that the requirement that $G$ be simple in Theorem 5.1 is essential. However, Sinclair [51] showed that one could eliminate this condition if one increases the lower bound on the minimum degree.

Theorem 5.9 Let $G$ be a 2-connected graph with minimum degree at least 5 . Then $G$ has a removable cycle.

This raises the question as to whether, by increasing the lower bound on circuit or cocircuit size in Question 5.3, one can guarantee the existence of a removable circuit in a 2-connected matroid. However, for all $r \geq 2$, the uniform matroid $U_{r, 2 r}$ is 2-connected and has no removable circuit although all its circuits and cocircuits have exactly $r+1$ elements. None of these uniform matroids is binary and the question as to whether the analogue of Theorem 5.9 holds for binary matroids remains open. Specifically, Goddyn and Jackson [19] asked the following: 
Question 5.10 Does there exist an integer $t$ such that every 2-connected binary matroid for which every cocircuit has size at least $t$ has a removable circuit?

Examples given earlier show that such an integer $t$ must exceed four, so the following is the natural first special case of the last question.

Question 5.11 If $M$ is a 2-connected binary matroid in which every cocircuit has size at least 5 , then does $M$ have a removable circuit?

Although the answer to Question 5.3 is negative, Goddyn and Jackson [19] were able to prove that 2-connected regular matroids do have removable circuits. More precisely, they proved the following result.

Theorem 5.12 Let $M$ be a 2-connected binary matroid in which every cocircuit has size at least 5 . If $M$ does not minors isomorphic to both $F_{7}$ and $F_{7}^{*}$, then $M$ has a circuit $C$ such that $M \backslash C$ is 2-connected and $r(M \backslash C)=r(M)$.

While the examples given above leave open the possibility that a binary 2-connected matroid with no small cocircuits may have a removable circuit, they suggest that, for matroids in general, a change in direction is needed. Mader's original result for a 2-connected graph $G$ includes the hypothesis that all vertex degrees are at least 4 . This implies that

$$
|E(G)| \geq 2|V(G)|
$$

Recall that the rank of a matroid is the size of a largest set that contains no circuit. Thus, the rank of the cycle matroid $M(G)$ of a graph $G$ is the number of edges in a spanning tree of $G$, that is, $|V(G)|-1$. Condition (5.1) implies that $M(G)$ satisfies the rank condition,

$$
|E| \geq 2 r(M)+2
$$

where we recall that the rank of a matroid is the size of a largest set that contains no circuit.

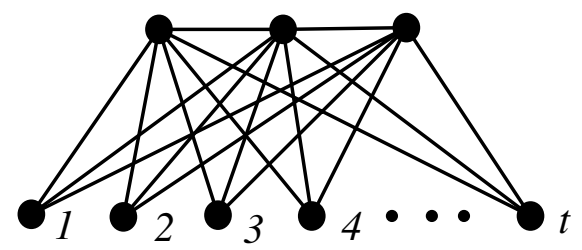

Figure 11. 
In view of Lemos's example and the fact that (5.2) is implied by Mader's original degree condition, one may guess that a 2-connected simple matroid $M$ satisfying (5.2) need not have a removable circuit. This turns out to be true although Lemos's example cannot be used to establish this since it does not satisfy (5.2). For an integer $t$ exceeding two, consider the graph $K_{3, t}^{\prime \prime}$, shown in Figure 11, that is obtained from $K_{3, t}$ by adding an edge from one of the degree- $t$ vertices to each of the other degree- $t$ vertices. The cycle matroid $M$ of this graph has no removable circuits since every circuit must meet a vertex of degree three. However,

$$
|E|=3 r(M)-4 \text {. }
$$

This prompts one to ask whether the existence of a removable circuit is guaranteed if one replaces (5.2) by a stronger condition of the same type. The next theorem [32] answers this question.

Theorem 5.13 Let $M$ be a 2-connected matroid with at least two elements and let $C^{\prime}$ be a largest circuit of $M$. If

$$
|E(M)| \geq 3 r(M)+3-c(M),
$$

then $M$ has a removable circuit $C$ that is disjoint from $C^{\prime}$ such that $r(M \backslash C)=$ $r(M)$. In particular, if $C^{\prime}$ spans $M$ and

$$
|E(M)| \geq 2 r(M)+2,
$$

then $M$ has a removable circuit.

Observe that when $M$ has a spanning circuit, condition (5.2) is sufficient to guarantee the existence of a removable circuit. In that case, the bound on $|E(M)|$ is sharp as one can see by taking $M=M(G)$ where $G$ is obtained from an $n$-cycle with $n \geq 2$ by replacing all but one of the edges by two edges in parallel. To see that the first bound on $|E(M)|$ in the last result is sharp, consider the cycle matroid of $K_{3, t}^{\prime \prime}$.

The next result follows by applying the last theorem to graphic matroids.

Corollary 5.14 Let $G$ be a 2-connected loopless graph and $C^{\prime}$ be a largest cycle in $G$. If

$$
|E(G)| \geq 3|V(G)|-c(G)
$$

then $G$ has a removable cycle $C$ that has no common edges with $C^{\prime}$. In particular, if $G$ is Hamiltonian and

$$
|E(G)| \geq 2|V(G)|
$$

then $G$ has a removable cycle. 
The assertion in the last sentence is easily deduced directly as follows. If $C^{\prime}$ is a Hamilton cycle in $G$, then, since $|E(G)| \geq 2|V(G)|$, we have $\left|E\left(G \backslash C^{\prime}\right)\right| \geq$ $\left|V\left(G \backslash C^{\prime}\right)\right|$. Hence $G \backslash C^{\prime}$ certainly has a cycle $C$. This cycle is clearly removable in $G$. In the case that $G$ is not Hamiltonian, the assertion of the corollary seems far less obvious. Theorem 5.13 can also be applied to the bond matroid of a 2-connected loopless graph $G$ in which case it gives a necessary condition for $G$ to have a bond $C^{*}$ such that $G / C^{*}$ is 2-connected and loopless.

To summarize what occurred above, we began with Mader's theorem for 2-connected graphs and tried to get a matroid analogue of it. While our initial attempts failed, eventually we obtained a rather loose analogue which is not only a new theorem for matroids but also a new theorem for graphs.

\section{Removing circuits from 3-connected matroids}

Although we have focussed so far on 2-connected matroids, Mader's theorem, with which we began this discussion, actually holds for $k$-connected graphs for all $k$. We have noted already that it is for $k$ in $\{2,3\}$ that Tutte's definition of $k$-connectedness for matroids has been most successfully analyzed. This leads us to ask whether the last result has an analogue for 3-connected matroids, although we have yet to define 3-connectedness for matroids. As a guide to how to do this, we look again at graphs.

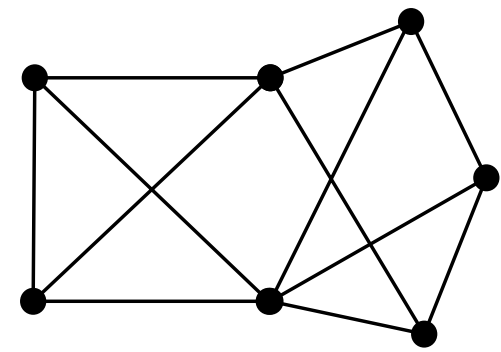

(a)

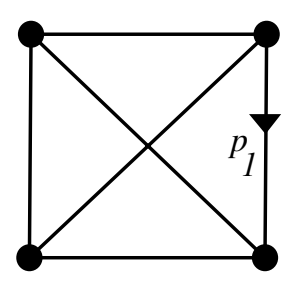

(b)

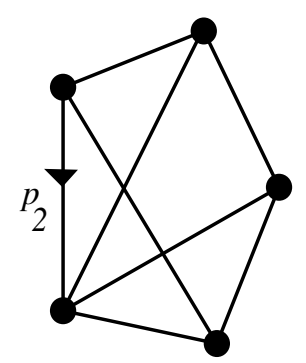

Figure 12. (a) $G$ (b) $G_{1}$ and $G_{2}$

The graph $G$ in Figure 12(a) is 2-connected but not 3-connected. Indeed, $G$ is the 2-sum of the graphs $G_{1}$ and $G_{2}$ shown in Figure $12(\mathrm{~b})$, that is, $G$ can be obtained from $G_{1}$ and $G_{2}$ by identifying the directed edges $p_{1}$ and $p_{2}$ and then deleting the resulting edge. The crucial observation here about $G$, $G_{1}$, and $G_{2}$ is that the edge sets of cycles of $G$ can be specified in terms of the cycles of $G_{1}$ and the cycles of $G_{2}$. Thus, the 2-sum operation for graphs generalizes to matroids as follows: let $M_{1}$ and $M_{2}$ be 2-connected matroids on disjoint ground sets $E_{1}$ and $E_{2}$ each of which has at least three elements and suppose that $p_{1} \in E_{1}$ and $p_{2} \in E_{2}$. The 2-sum $M_{1} \oplus_{2} M_{2}$ of $M_{1}$ and $M_{2}$ with respect to $p_{1}$ and $p_{2}$ is the matroid with ground set $\left(E_{1}-p_{1}\right) \cup\left(E_{2}-p_{2}\right)$ whose circuits are

- all circuits of $M_{1}$ avoiding $p_{1}$; 
- all circuits of $M_{2}$ avoiding $p_{2}$; and

- all sets of the form $\left(C_{1}-\left\{p_{1}\right\}\right) \cup\left(C_{2}-\left\{p_{2}\right\}\right)$ where $C_{i}$ is a circuit of $M_{i}$ containing $p_{i}$.

A 2-connected matroid is 3-connected if it cannot be written as a 2-sum of two other matroids. This definition is able to simultaneously generalize the corresponding graph notion and to incorporate invariance under duality. In particular, if $G$ is a graph without isolated vertices and with at least four vertices, then the cycle matroid $M(G)$ of $G$ is 3-connected if and only if $G$ is simple and 3-connected. An arbitrary matroid is 3-connected if and only if its dual is 3-connected.

The next theorem, which was proved by Lemos and Oxley [33], is an analogue of Theorem 5.13 for 3-connected matroids.

Theorem 6.1 Let $M$ be a 3-connected matroid and $C^{\prime}$ be a largest circuit of M. If

$$
|E(M)| \geq \begin{cases}3 r(M)+1 & \text { when } c(M)=r(M)+1 \\ 4 r(M)+1-c(M) & \text { otherwise, }\end{cases}
$$

then $M$ has a circuit $C$ that is disjoint from $C^{\prime}$ such that $M \backslash C$ is 3-connected and $r(M \backslash C)=r(M)$.

As with Theorem 5.13, we can apply the last result to graphs to obtain a new graph result.

Corollary 6.2 Let $G$ be a simple 3-connected graph and $C^{\prime}$ be a largest cycle of $G$. Suppose that

$$
|E(G)| \geq \begin{cases}3|V(G)|-2 & \text { if } G \text { is Hamiltonian } \\ 4|V(G)|-3-c(G) & \text { otherwise }\end{cases}
$$

Then $G$ has a cycle $C$ that has no common edges with $C^{\prime}$ such that $G \backslash C$ is 3-connected.

\section{Minors and infinite antichains}

Minors are basic substructures of graphs. Indeed, one of the best-known theorems in graph theory is Kuratowski's Theorem [28, 60] characterizing planar graphs.

Theorem 7.1 A graph $G$ is planar if and only if it has no minor isomorphic to $K_{5}$ or $K_{3,3}$. 
This section begins with a discussion of such excluded-minor theorems for graphs and matroids. It concludes by considering a problem for matroid minors that is motivated by a recent celebrated graph result. Once again, the interaction between graph theory and matroid theory should be evident.

It was noted in Section 2 that, for a graphic matroid $M$, all single-element deletions and all single-element contractions are also graphic. It follows that every minor of $M$ is graphic, and we say that the class of graphic matroids is minor-closed.

In Section 2, we noted that the matroid $U_{2,4}$ is not binary and that every graphic matroid is binary. It follows that $U_{2,4}$ is not graphic, though it is just as easy to show this directly. Every single-element deletion of $U_{2,4}$ is isomorphic to $U_{2,3}$, that is, to $M\left(K_{3}\right)$. Since $U_{2,4}^{*} \cong U_{2,4}$, we deduce that every single-element contaction of $U_{2,4}$ is isomorphic to $U_{1,3}$, that is, to $M\left(C_{3}^{*}\right)$ where $C_{3}^{*}$ is the graph consisting of two vertices joined by three edges. We conclude that $U_{2,4}$ is an excluded minor for the class of graphic matroids because it is not in the class, yet all of its proper minors are in the class. Another excluded minor for the class is the Fano matroid: every single-element deletion of $F_{7}$ is isomorphic to $M\left(K_{4}\right)$, while all of its single-element contractions are isomorphic to $M\left(C_{3}^{2}\right)$, where $C_{3}^{2}$ is the graph obtained from a 3 -cycle by replacing every edge by two parallel edges. Since the planar duals of $K_{4}$ and $C_{3}^{2}$ are $K_{4}$ and $K_{2,3}$, respectively, it follows that every proper minor of $F_{7}^{*}$ is also graphic. The following excluded-minor characterization of the class of graphic matroids, which was proved by Tutte [54], is a generalization of Theorem 7.1.

Theorem 7.2 A matroid is graphic if and only if it has no minor isomorphic to any of the matroids $U_{2,4}, F_{7}, F_{7}^{*}, M^{*}\left(K_{5}\right)$, and $M^{*}\left(K_{3,3}\right)$.

On combining this theorem with its dual and using Kuratowski's Theorem, we obtain the following result.

Theorem 7.3 A matroid is isomorphic to the cycle matroid of a planar graph if and only if it has no minor isomorphic to any of the matroids $U_{2,4}, F_{7}, F_{7}^{*}$, $M^{*}\left(K_{5}\right), M\left(K_{5}\right), M^{*}\left(K_{3,3}\right)$, and $M\left(K_{3,3}\right)$.

Next we shall show that, for every field $\mathbb{F}$, the class of $\mathbb{F}$-representable matroids is minor-closed. To see this, suppose that $M=M[A]$ for some matrix $A$ over $\mathbb{F}$. If $e$ is an element of $M$, then $M \backslash e$ is represented by the matrix that is obtained from $A$ by deleting the column of $A$ labelled by $e$. To show that $M / e$ is $\mathbb{F}$-representable, we argue as follows. If the column labelled by $e$ is zero, then $M[A] / e=M[A] \backslash e$, and so the contraction of $e$ is certainly $\mathbb{F}$-representable. If the column labelled by $e$ is non-zero, then we choose a non-zero entry of this column, say the entry in row $i$. We now add suitable multiples of row $i$ to the other rows of $A$ to produce a matrix $A^{\prime}$ in which the column labelled by $e$ is the $i$ th unit vector. It is not difficult to check that $M\left[A^{\prime}\right]=M[A]$. The contraction $M\left[A^{\prime}\right] / e$ is represented by the matrix that is 
obtained from $A^{\prime}$ by deleting row $i$ and column $e$. We conclude that the class of $\mathbb{F}$-representable matroids is minor-closed.

It was noted in Section 2 that the dual of every $\mathbb{F}$-representable matroid is F-representable. Therefore the dual of every excluded minor for the class of $\mathbb{F}$-representable matroids is also an excluded minor. Now let $\mathbb{F}=G F(2)$. The excluded-minor characterization of the class of binary matroids was proved by Tutte [56]. We have already identified the only excluded minor for this class.

Theorem 7.4 A matroid is binary if and only if it has no minor isomorphic to $U_{2,4}$.

The classes of $G F(3)$-representable and $G F(4)$-representable matroids are called, respectively, the classes of ternary and quaternary matroids. Proving the excluded-minor characterizations of these classes was far more difficult than proving Theorem 7.4. Ralph Reid announced the result for ternary matroids in 1971 but never published a proof. The first published proofs are due to Bixby [1] and Seymour [49].

Theorem 7.5 A matroid is ternary if and only if it has no minor isomorphic to any of the matroids $U_{2,5}, U_{3,5}, F_{7}$, and $F_{7}^{*}$.

The next theorem was even more difficult to obtain than the last. It was proved by Geelen, Gerards, and Kapoor [17]. The non-Fano matroid, $F_{7}^{-}$, which is shown in Figure 5(a), is also equal to the matroid that is represented over $G F(3)$ by the matrix whose columns consist of all 3 -tuples of zeros and ones except $(0,0,0)^{T}$. The matroid $P_{6}$ is represented geometrically by six points placed in the plane so that exactly one 3 -element subset is collinear. The matroids $P_{8}$ and $P_{8}^{=}$are represented over $G F(3)$ and $G F(5)$ by the matrices $\left[I_{4} \mid A_{1}\right]$ and $\left[I_{4} \mid A_{2}\right]$ where $A_{1}$ and $A_{2}$ are, respectively,

$$
\left[\begin{array}{cccc}
0 & 1 & 1 & -1 \\
1 & 0 & 1 & 1 \\
1 & 1 & 0 & 1 \\
-1 & 1 & 1 & 0
\end{array}\right] \text { and }\left[\begin{array}{cccc}
1 & 1 & 1 & 1 \\
1 & 1 & -2 & -1 \\
1 & -1 & 0 & -1 \\
1 & 2 & 1 & 0
\end{array}\right]
$$

All of $P_{6}, P_{8}$, and $P_{8}^{=}$are isomorphic to their duals. Next we describe a very attractive geometric representation for the matroid $P_{8}$. Begin with a cube in 3space, its eight vertices being the elements of the matroid. Then, in the plane of the top face, rotate the face through $45^{\circ}$ about its centre. The resulting configuration represents $P_{8}$, its circuits being all sets of four coplanar points and all sets of five points no four of which are coplanar. We obtain $P_{8}^{=}$from $P_{8}$ by relaxing the top and bottom faces, both of which are circuit-hyperplanes of the twisted cube $P_{8}$.

Theorem 7.6 A matroid is quaternary if and only if it has no minor isomorphic to any of the matroids $U_{2,6}, U_{4,6}, P_{6}, F_{7}^{-},\left(F_{7}^{-}\right)^{*}, P_{8}$, and $P_{8}^{=}$. 
The last result verifies a special case of a conjecture of Rota [47], which was made after Theorems 7.4 and 7.5 had been announced. This conjecture is probably the most important unsolved problem in the study of representable matroids. It remains open for all $q \geq 5$.

Conjecture 7.7 If $q$ is a prime power, then the set of excluded minors for representability over $G F(q)$ is finite.

Not only are the lists of excluded minors in the last three matroid theorems finite, but so too are the lists in Theorems 7.2 and 7.3. In each case, there are only finitely many obstructions to the specified matroid property. Wagner conjectured that every minor-closed class of graphs has a finite list of excluded minors (see, for example, [42, p. 155]). We call a set of graphs or a set of matroids an antichain if no member of the set is isomorphic to a minor of another member of the set. As the culmination of a long series of difficult papers, Robertson and Seymour [46] proved Wagner's conjecture by establishing the following result.

Theorem 7.8 There is no infinite antichain of graphs.

An immediate consequence of this theorem is the following generalization of Kuratowski's Theorem.

Theorem 7.9 If $S$ is a surface, then there is a set $\left\{G_{1}, G_{2}, \ldots, G_{n}\right\}$ of graphs such that an arbitrary graph $G$ can be embedded in $S$ if and only if $G$ has none of $G_{1}, G_{2}, \ldots, G_{n}$ as a minor.

Given the intimate links between graphs and matroids, it is natural to ask whether Theorem 7.8 extends to matroids. But, even before that theorem was proved, it was known that infinite antichains of matroids do exist (see, for example, $[3, \mathrm{p} .155])$. The set $\left\{C_{3}, C_{4}, C_{5}, \ldots\right\}$ of cycles with at least three vertices is clearly infinite, and no member of this set is isomorphic to a subgraph of another. We can use this set of graphs, or, indeed, any infinite set of simple graphs with the last property, to build an infinite antichain of matroids as follows: embed each $C_{n}$ in the plane so that no three vertices are collinear, viewing its vertices as points of a rank-3 matroid and its edges as lines of the matroid. Then add one extra point on each line to get a matroid $M_{n}$ that consists of a ring of $n$ three-point lines. It is not difficult to check that $\left\{M_{3}, M_{4}, M_{5}, \ldots\right\}$ is an infinite antichain of matroids. For each prime power $q$, the points of the projective plane $P G(2, q)$ are the points of a rank-3 matroid in which the circuits are all sets of three collinear points and all sets of four points with no three collinear. Another infinite antichain of matroids is $\{P G(2, p)$ : $p$ is prime $\}$.

Let $\mathcal{M}_{a, b, c}$ be the class of matroids such that, by deleting at most $a$ elements and contracting at most $b$ elements, one obtains a matroid in which each 
component has at most $c$ elements. Thus each of the infinite antichains above is contained in $\mathcal{M}_{0,3,1}$. Ding, Oporowski, and Oxley [10] determined precisely when $\mathcal{M}_{a, b, c}$ contains an infinite antichain.

Theorem 7.10 The class $\mathcal{M}_{a, b, c}$ contains an infinite antichain if and only if none of the following holds.

(i) $\min \{a, b\}=0$ and $\max \{a, b\}=1$;

(ii) $\min \{a, b\}=0, \max \{a, b\}=2$, and $c=2$; and

(iii) $\max \{a, b\} \leq 2$ and $c \leq 1$.

By contrast, insisting on representability over a fixed finite field completely changes the result.

Theorem 7.11 For all prime powers $q$ and all non-negative integers $a, b$, and $c$, there is no infinite antichain in $\mathcal{M}_{a, b, c}$ in which all the members are $G F(q)$-representable.

Yet another infinite antichain of matroids was constructed by Lazarson [29] to establish the following result which contrasts strikingly with Conjecture 7.7.

Theorem 7.12 If $\mathbb{F}$ is a field of characteristic zero, then the set of excluded minors for $\mathbb{F}$-representability is infinite.

We now know that Theorem 7.8 fails for the class of all matroids. Since each $M_{i}$ constucted at the top of this page is $\mathbb{R}$-representable, it also fails for the class of $\mathbb{R}$-representable matroids. A problem that is currently attracting much research attention is whether Theorem 7.8 can be generalized to the class of binary matroids. More generally, we have the following:

Question 7.13 Let $q$ be a prime power. Is there an infinite antichain of $G F(q)$-representable matroids?

This question and Rota's conjecture appear quite similar. But they are different. Question 7.13 asks about the existence of an infinite set of matroids within the class of $G F(q)$-representable matroids such that no member of the set is a minor of another member of the set. Conjecture 7.7 asserts that, within the set of matroids that are not $G F(q)$-representable, there are only finitely many minor-minimal members. Indeed, it is not clear how settling one of Question 7.13 and Conjecture 7.7 would assist in settling the other. We have remarked already on the importance of Conjecture 7.7. In the context of this paper, describing how graphs and matroids interact, Question 7.13 is the most important open problem for it seeks to obtain a natural matroid extension of what is probably the most difficult theorem ever proved in graph theory. 


\section{Branch-width and infinite antichains}

Recently, Geelen, Gerards, and Whittle [18] have proved an important partial result towards resolving Question 7.13 in the negative. One interesting feature of their result is that it can be used to give an alternative proof of the corresponding result for graphs, which was an important early step [43] in the derivation of Theorem 7.8. To explain this new matroid result, we shall need to define branch-width for matroids. This is an analogue of its namesake for graphs. The latter is closely related to the more widely used notion of tree-width. Robertson and Seymour [45] proved that a family of graphs has bounded branch-width if and only if it has bounded tree-width. Although neither of these notions has yet been defined here, we mention this result for the reader who is already familiar with the concept of tree-width. In order to simplify the somewhat complex discussion to follow, we shall restrict attention to branch-width.

Before defining branch-width for matroids, we return to 2- and 3-connectedness for matroids and consider what these concepts mean in terms of the rank function. A matroid $M$ is 2-connected if and only if, for every partition $\{X, Y\}$ of $E(M)$, there is a circuit meeting both $X$ and $Y$, or, equivalently,

$$
r(X)+r(Y)-r(M) \geq 1 \text {. }
$$

In other words, $M$ is 2-connected if and only if it has no 1-separation where, for a positive integer $k$, a partition $\{X, Y\}$ of the ground set of a matroid $M$ is a $k$-separation of $M$ if

$$
\begin{gathered}
r(X)+r(Y)-r(M)+1 \leq k \text { and } \\
\min \{|X|,|Y|\} \geq k .
\end{gathered}
$$

For $n \geq 2$, a matroid $M$ is $n$-connected if there is no $k$ in $\{1,2, \ldots, n-1\}$ such that $M$ has a $k$-separation. This definition implies, for example, that an $n$-connected matroid with at least $2 n-2$ elements has no circuits or cocircuits of size less than $n$. The Fano and non-Fano matroids, which are shown in Figure 5, are both examples of 3-connected matroids that are not 4-connected.

The quantity $r(X)+r(Y)-r(M)+1$, which, following Tutte [58], we denote by $\xi(M ; X, Y)$, also features prominently in the definition of branchwidth. The degree-one vertices of a tree are called leaves. A ternary tree is a tree in which all vertices except the leaves have degree three. A branchdecomposition of a matroid $M$ consists of a ternary tree $T$ with exactly $|E(M)|$ leaves together with a labelling of these leaves so that each is labelled by a different element of $E(M)$. For each edge $e$ of such a branch-decomposition $T$ of $M$, the graph $T \backslash e$ has exactly two components. Thus the set of leaves of $T$, and hence $E(M)$, is partitioned into two subsets $X$ and $Y$, say. The width of the edge $e$ is defined to be $\xi(M ; X, Y)$ and the width of $T$ is the maximum of the widths of the edges of $T$. If $|E(M)| \geq 2$, the branch-width bw $(M)$ of $M$ 
is the minimum, over all such labelled ternary trees $T$, of the width of $T$. If $|E(M)| \leq 1$, then its branch-width is zero.

The next result [6] summarizes some basic properties of branch-width.

Proposition 8.1 Let $M$ be a matroid. Then

(i) $b w\left(M^{*}\right)=b w(M)$;

(ii) $b w(N) \leq b w(M)$ for every minor $N$ of $M$;

(iii) if $|E(M)| \geq 3$ and $e \in E(M)$, then both bw $(M \backslash e)$ and bw $(M / e)$ are in $\{b w(M)-1, b w(M)\}$;

(iv) if $M$ has a component with at least two elements, then bw $(M)$ is the maximum of the branch-widths of its components;

(v) bw $(M) \leq 2$ if and only if every component of $M$ is isomorphic to a series-parallel network; and

(vi) if $M$ is $n$-connected and $n \geq 3$, then bw $(M) \geq n$ if and only if $|E(M)| \geq$ $3 n-5$.

The last part of this proposition says, loosely speaking, that if a matroid is highly connected and has a lot of elements, then its branch-width is also high. To try to convey some intuition for what it means for branch-width to be small, we observe that a vector matroid has small branch-width if and only if it can be obtained from small matroids by sticking these together in a tree-like structure across subspaces of the underlying vector space.

Theorem 7.8 and Proposition 8.1(v) imply that there is no infinite antichain of matroids of branch-width at most two. The antichain of rank-3 matroids obtained from $\left\{C_{3}, C_{4}, C_{5}, \ldots\right\}$ following Theorem 7.9 implies that there are infinite antichains of representable matroids of branch-width at most four. Geelen, Gerards, and Whittle [18] improved this by showing that infinite antichains of representable matroids arise within the class of matroids of branch-width three.

Theorem 8.2 There is an infinite antichain of matroids each of which has branch-width three and is representable over all infinite fields.

The main result of Geelen, Gerards, and Whittle [18] is the following theorem which is an important step towards resolving Question 7.13. This theorem extends Theorem 7.11 since, by Proposition 8.1, every member of $\mathcal{M}_{a, b, c}$ has branch-width at most $a+b+c$.

Theorem 8.3 For all prime powers $q$ and all positive integers $n$, there is no infinite antichain of $G F(q)$-representable matroids each having branch-width at most $n$. 
The techniques developed to prove the last result are very interesting and will be discussed in Section 10. In addition, the last theorem can be used to prove the following graph result of Robertson and Seymour [43], a significant early step in the proof of Theorem 7.8.

Theorem 8.4 For all positive integers $n$, there is no infinite antichain of graphs all having branch-width at most $n$.

To this point, the notion of branch-width has only been defined here for matroids although we have briefly mentioned the corresponding notion for graphs. One may hope that these two notions coincide but this has yet to be proved. Before proceeding further with this discussion, we shall define branchwidth for graphs. The definition mimics that of branch-width for matroids but replaces a matroid $M$ and its connectivity function $\xi(M ; X, Y)$ by a graph $G$ and its connectivity function $\eta(G ; X, Y)$. The latter is defined, when $\{X, Y\}$ is a partition of $E(G)$, to be the number of vertices common to $G[X]$ and $G[Y]$, the subgraphs of $G$ induced by the edges in $X$ and $Y$. Now

$$
\xi(M(G) ; X, Y)=\eta(G ; X, Y)+\omega(G)-\omega(G[X])-\omega(G[Y])+1
$$

where $\omega(H)$ is the number of components of a graph $H$. Thus if $\beta(G)$ is the branch-width of a connected graph $G$, then

$$
b w(M(G)) \leq \beta(G)
$$

The last inequality is strict, for example, when $G$ is the graph that is obtained from $K_{2}$ by adding a loop at each vertex. In that case, $b w(M(G))=1$ and $\beta(G)=2$. In addition, the inequality fails when $G$ consists of the disjoint union of $k$ copies of $K_{2}$ for some $k \geq 2$. In that case, $\beta(G)=0$ but bw $(M(G))=$ 1. The examples in which equality fails to hold in (8.2) seem very specialized. Indeed, Geelen, Gerards, and Whittle (private communication, 2000) have proposed the following conjecture, towards which some partial results were previously established by Dharmatilake [5].

Conjecture 8.5 Let $G$ be a graph that cannot be obtained from a forest by adjoining loops. Then

$$
b w(M(G))=\beta(G)
$$

To obtain Theorem 8.4 from Theorem 8.3, one combines (8.2) with a wellknown consequence of a result of Higman [21] that if there is no infinite antichain within a class $\mathcal{G}$ of connected finite graphs, then there is no infinite antichain within the class of finite graphs for which every component is in $\mathcal{G}$. 


\section{The implications of large branch-width}

Theorem 8.3 is a significant step in trying to generalize to matroids one of the major achievements of the Graph-Minors Project, Theorem 7.8. An important part of the proof of the last result involves determining what happens in a graph when the branch-width is large. The $n \times n$ grid is the graph with vertex-set $\{(i, j): i, j \in\{1,2, \ldots, n\}\}$ such that $(i, j)$ and $\left(i^{\prime}, j^{\prime}\right)$ are adjacent if and only if $\left|i-i^{\prime}\right|+\left|j-j^{\prime}\right|=1$. The next result follows immediately by combining two theorems of Robertson and Seymour [44, 45].

Theorem 9.1 For each positive integer $n$, there is an integer $k(n)$ such that every graph of branch-width at least $k(n)$ has a minor isomorphic to the $n \times n$ grid.

A matroid generalization of the last result has recently been conjectured by Johnson, Robertson, and Seymour. In order to state it, we shall require some more definitions. The cycle and bond matroids of a graph have already been defined. There is another interesting, but less-well-studied, matroid that arises from a graph $G$. The cycle matroid of $G$ can be defined as the graph on $E(G)$ for which the circuits are the edge-sets of all subgraphs that are subdivisions of a loop. The bicircular matroid of $G$ is the matroid on $E(G)$ in which the circuits are the edge-sets of all subgraphs that are subdivisions of one of the following three graphs: two vertices joined by three edges; two loops at the same vertex; two loops at distinct vertices that are joined by a single edge. The $n \times n$ griddle is the bicircular matroid of the $n \times n$ grid. The $n \times n$ girdle is the dual of the $n \times n$ griddle. Johnson, Robertson, and Seymour's [26] conjecture is as follows.

Conjecture 9.2 For each positive integer $n$, there is an integer $k(n)$ such that every matroid of branch-width at least $k(n)$ has a minor isomorphic to one of $U_{n, 2 n}$, the cycle matroid of the $n \times n$ grid, the $n \times n$ griddle, or the $n \times n$ girdle.

By using Theorems 7.4 and 7.5, it is straightforward to check that none of $U_{3,6}$, the $3 \times 3$ griddle, and the $3 \times 3$ girdle is binary or ternary. Thus, for example, the following is the specialization of the last conjecture to binary matroids.

Conjecture 9.3 For each positive integer $n$, there is an integer $k(n)$ such that every binary matroid of branch-width at least $k(n)$ has a minor isomorphic to the cycle matroid of the $n \times n$ grid.

If true, this conjecture would generalize Theorem 9.1 provided Conjecture 8.5 is also true. Some progress has been made towards Conjecture 9.3 by Johnson, Robertson, and Seymour [25]. 


\section{Some proof outlines}

In this section, we provide some of the details of the proofs of Theorems 7.9, 8.3, and 8.2. Theorem 7.8 occurs in the twentieth of a sequence of long and difficult papers. An outline of the proof appears in [7, Chapter 12]. By contrast, the proof of Theorem 7.9, Kuratowski's Theorem for arbitrary surfaces, is far more accessible and, next, we indicate the main steps in this proof. By Theorem 8.4, whose proof is relatively short, a counterexample to Theorem 7.9 would contain graphs of arbitrarily high branch-width. By Theorem 9.1, of which a short self-contained proof has been given by Diestel, Jensen, Gorbunov, and Thomassen [8], such graphs contain arbitrarily large grid-minors. Finally, Thomassen [53] has given a short proof that a minor-minimal graph that does not embed on a surface does not contain a large grid minor.

There are three main tools in Robertson and Seymour's proof of Theorem 8.4:

(i) a lemma on rooted trees that generalizes Kruskal's theorem [27] that, under the relation of topological containment, there is no infinite antichain of trees;

(ii) Menger's Theorem; and

(iii) a theorem of Thomas [52] on linked tree-decompositions, that is, treedecompositions with a certain Menger-like property.

Geelen, Gerards, and Whittle's proof of Theorem 8.3 has the same basic structure. It uses (i) together with a matroid generalization of Menger's Theorem due to Tutte [57], and a new linked-branch-decomposition theorem. We shall concentrate here on the last two of these and refer the reader to [43] or [18] for the details of (i).

Tutte's matroid generalization of Menger's Theorem is a relatively old result whose significance appears not to have been appreciated until relatively recently. In addition to being used by Geelen, Gerards and Whittle, it also plays a role in the proof of Johnson, Robertson, and Seymour's partial result towards Conjecture 9.3. We state the theorem next and then derive Menger's Theorem as a corollary of it.

Theorem 10.1 Let $S$ and $T$ be disjoint non-empty subsets of a matroid $M$. Then the minimum value of $\xi(M ; X, Y)$ over all subsets $X$ and $Y$ such that $X \supseteq S$ and $Y \supseteq T$ equals the maximum value of $\xi(N ; S, T)$ over all minors $N$ of $M$ having ground set $S \cup T$.

Corollary 10.2 Let $s$ and $t$ be distinct non-adjacent vertices in a graph $G$. Then the minimum number of vertices needed to separate $s$ from $t$ equals the maximum number of internally disjoint paths joining $s$ and $t$. 
Proof Suppose that the minimum number of vertices needed to separate $s$ from $t$ is $m$. The non-trivial part of the corollary is to establish that $G$ has $m$ internally disjoint paths joining $s$ and $t$. Clearly this holds if $m \leq 1$. Thus we may assume that $m \geq 2$. There is no loss of generality in assuming that $G$ is connected. Let $S$ and $T$ be the sets of edges incident with $s$ and $t$, respectively. Then $G[S]$ and $G[T]$ are connected and it is not difficult to check that the minimum value of $\xi(M(G) ; X, Y)$ can be achieved by some $X$ and $Y$ such that $G[X]$ and $G[Y]$ are connected. For this choice of $X$ and $Y$, we have, by $(8.1)$, that $\eta(G ; X, Y)=\xi(M(G) ; X, Y)$. As $G[X]$ and $G[Y]$ must have at least $m$ common vertices, we deduce that $\xi(M(G) ; X, Y) \geq m$. Since it is not difficult to construct $X^{\prime}$ and $Y^{\prime}$ with $X^{\prime} \supseteq S$ and $Y^{\prime} \supseteq T$ such that $\eta\left(G ; X^{\prime}, Y^{\prime}\right)=m$, we deduce, by $(8.1)$, that $\xi\left(M(G) ; X^{\prime}, Y^{\prime}\right) \leq m$. Since $\xi(M(G) ; X, Y) \leq \xi\left(M(G) ; X^{\prime}, Y^{\prime}\right)$, we conclude that $\xi(M(G) ; X, Y)=m$. Thus, by Theorem 10.1, $G$ has a minor $H$ with edge set $S \cup T$ such that $\xi(M(H) ; S, T)=m$. Thus, since $H[S]$ and $H[T]$ are both connected, $\omega(H) \leq 2$ and, by $(8.1), m=\eta(H ; S, T)+\omega(H)-1$. If $\omega(H)=2$, then $\eta(H ; S, T)=0$ and we obtain the contradiction that $m=1$. Thus $\omega(H)=1$, so $\eta(H ; S, T)=m$, that is, $H[S]$ and $H[T]$ have $m$ common vertices. Since $S$ and $T$ must be the sets of edges incident with $s$ and $t$ in $H$, it follows that $H$ has $m$ internally disjoint paths joining $s$ and $t$. Therefore so does $G$.

The third main tool in the proof of Theorem 8.3 is the analogue of Thomas's linked-tree-decomposition result. Geelen, Gerards, and Whittle were able to avoid many of the technicalities of Thomas's proof by using branch-width rather than tree-width. In addition, they took advantage of the similarities between the functions $\xi(M ; X, Y)$ and $\eta(G ; X, Y)$. Since $Y$ is the complement of $X$, each of $\xi$ and $\eta$ can be viewed as a function of the variable $X$. As such, each is both submodular and symmetric where, in general, a function $\lambda$ on the set of subsets of a set $E$ is submodular if $\lambda(A)+\lambda(B) \geq \lambda(A \cup B)+\lambda(A \cap B)$ for all $A, B \subseteq E$; and $\lambda$ is symmetric if $\lambda(A)=\lambda(E-A)$ for all $A \subseteq E$. Submodular functions have arisen earlier in the paper, though not explicitly, for a basic property of the rank function of a matroid is that it is submodular.

One can define a branch-decomposition of an arbitrary symmetric submodular function $\lambda$ on a set $E$ in just the same way as it was defined for $\xi$. A subset $X$ of $E$ is displayed by a branch-decomposition $T$ if there is an edge $e$ of $T$ such that the sets of vertex labels that occur (on the leaves of $T$ ) in the two components of $T \backslash e$ are $X$ and $E-X$. The width of such an edge is the common value of $\lambda(X)$ and $\lambda(E-X)$. This leads to the definition of the branch-width of $\lambda$. Let $f$ and $g$ be two edges in a branch-decomposition $T$ of $\lambda$ and let $F$ and $G$ be, respectively, the component of $T \backslash f$ avoiding $g$ and the component of $T \backslash g$ avoiding $f$. Each edge on the shortest path $P$ in $T$ containing $f$ and $g$ displays a subset of $E$ that contains $F$ and avoids $G$. Thus the widths of the edges of $P$ are upper bounds on $\lambda(F, G)$, the minimum value of $\lambda(X)$ taken over all $X$ such that $F \subseteq X \subseteq E-G$. We say that 
$F$ and $G$ are linked if $\lambda(F, G)$ actually equals the minimum of the widths of the edges of $P$. A branch-decomposition is linked if all of its edge pairs are linked. Such a branch-decomposition enables one to use Tutte's matroid form of Menger's Theorem, for it means that there will be no small separations between two disjoint displayed sets other than those that can be seen from the branch-decomposition. Geelen, Gerards and Whittle's result is the following.

Theorem 10.3 An integer-valued symmetric submodular function that has branch-width $n$ has a linked branch-decomposition of width $n$.

To conclude this section, we shall prove Theorem 8.2. The proof will be geometric and will involve a fundamental class of matroids called spikes. Geometrically, an $n$-spike with tip consists of $n$ three-point lines all passing through a common point but otherwise placed as freely as possible in rank- $n$ space. More formally, for $n \geq 3$, a rank- $n$ matroid $M$ is an $n$-spike with tip $p$ if it satisfies the following two conditions:

(a) $E(M)$ is the union of $n$ three-element circuits, $L_{1}, L_{2}, \ldots, L_{n}$, all of which contain the element $p$; and

(b) for all $k$ in $\{1,2, \ldots, n-1\}$, the union of any $k$ of $L_{1}, L_{2}, \ldots, L_{n}$ has rank $k+1$.

Thus, for example, each of $F_{7}^{-}$and $F_{7}$ in Figure 5 is a 3 -spike with tip 1 . In general, if $M$ is an $n$-spike with tip $p$, then

(i) $\left(L_{i} \cup L_{j}\right)-\{p\}$ is a circuit of $M$ for all distinct $i$ and $j$;

(ii) apart from $L_{1}, L_{2}, \ldots, L_{n}$ and those sets listed in (i), every non-spanning circuit of $M$ avoids $p$, is a circuit-hyperplane, and contains a unique element from each of $L_{1}-\{p\}, L_{2}-\{p\}, \ldots, L_{n}-\{p\}$;

(iii) $M / p$ can be obtained from an $n$-element circuit by replacing each element by two elements in parallel; and

(iv) if $\{x, y\}=L_{i}-\{p\}$ for some $i$, then each of $M \backslash p / x$ and $(M \backslash p \backslash x)^{*}$ is an $(n-1)$-spike with tip $y$.

Spikes play an important role in matroid structure theory and will be discussed further in the next section. Sometimes spikes are considered with their tips deleted (and still called spikes) because such matroids equal their duals. We show next how spikes can be used to prove Theorem 8.2.

Proof of Theorem 8.2 From property (iii) of spikes and Proposition 8.1(v), it follows that, for $n \geq 3$, every $n$-spike has branch-width three. We complete the proof by identifying a special class of $n$-spikes. 
For $n \geq 4$, let $M_{n}$ be the $n$-spike with tip $p$ such that $L_{i}=\left\{p, a_{i}, b_{i}\right\}$ for all $i$ and $M_{n}$ has exactly two circuit-hyperplanes, namely $\left\{a_{1}, a_{2}, \ldots, a_{n}\right\}$ and $\left\{b_{1}, b_{2}, \ldots, b_{n}\right\}$. We shall show that $\left\{M_{n}: n \geq 5\right\}$ is an antichain in which all the members are representable over every infinite field.

To see that $\left\{M_{n}: n \geq 5\right\}$ is an antichain, we shall consider the minors of some $M_{n}$ with $n \geq 6$. To produce a matroid of lower rank, we may assume that some element of $M_{n}$ is contracted. But, from (iii) above, contracting the tip produces a matroid that does not have any $M_{k}$ as a minor. By symmetry, all other single-element contractions of $M_{n}$ are isomorphic to $M_{n} / a_{1}$. The last matroid is obtained from an $(n-1)$-spike by adding $b_{1}$ in parallel with the tip. Thus if some $M_{k}$ is a proper minor of $M_{n}$, then it is a minor of the $(n-1)$ spike $M / a_{1} \backslash b_{1}$. But the last matroid has a unique circuit-hyperplane, namely $\left\{a_{2}, a_{3}, \ldots, a_{n}\right\}$. Thus every proper spike-minor of $M_{n}$ of rank at least five has at most one circuit-hyperplane, and $\left\{M_{n}: n \geq 5\right\}$ is indeed an antichain.

Next we describe geometrically how to construct a matrix that represents $M_{n}$ over an arbitrary infinite field $\mathbb{F}$. For each vector in $V(n, \mathbb{F})$ the $n$-dimensional vector space over $\mathbb{F}$, we shall consider the corresponding point of the projective space $P G(n-1, \mathbb{F})$. This will enable us to argue geometrically. For each $i$ in $\{1,2, \ldots, n-1\}$, let $b_{i}$ correspond to the $i$ th unit vector in $V(n, \mathbb{F})$. Let $a_{n}, p$, and $b_{n}$ correspond, respectively, to the $n$th unit vector, the all-ones vector, and the vector with zero as its last entry and every other entry equal to one. Then $\left\{b_{1}, b_{2}, \ldots, b_{n}\right\}$ is a circuit in the resulting matroid. For each $i$ in $\{1,2, \ldots, n-1\}$, we now need to add $a_{i}$ to the line spanned by $b_{i}$ and $p$ so that $\left\{a_{1}, a_{2}, \ldots, a_{n}\right\}$ and $\left\{b_{1}, b_{2}, \ldots, b_{n}\right\}$ are the only circuits of the form $\left\{d_{1}, d_{2}, \ldots, d_{n}\right\}$ with $d_{i}$ in $\left\{a_{i}, b_{i}\right\}$ for all $i$. If we add $a_{1}, a_{2}, \ldots, a_{n-1}$ one at a time, then, at each stage, there are only finitely many points on the line spanned by $p$ and $b_{i}$ that, if they were used for $a_{i}$ would create unwanted circuits. (Equivalently, there are only finitely many 1-dimensional subspaces of $V(n, \mathbb{F})$ in the 2-dimensional subspace spanned by $p$ and $b_{i}$ such that, if $a_{i}$ were chosen in one of them, unwanted circuits would be created.) By choosing $a_{i}$ to avoid these points, which can certainly be done since $\mathbb{F}$ is infinite, we avoid all such unwanted circuits. Since $a_{n}$ was chosen at the outset, a little extra care must be employed in placing $a_{n-2}$. Its placement will immediately determine $a_{n-1}$ since we want $\left\{a_{1}, a_{2}, \ldots, a_{n}\right\}$ to be a circuit. This creates additional restrictions on the placement of $a_{n-2}$ since we need to avoid not only unwanted circuits involving $a_{n-2}$ but also unwanted circuits involving the resulting $a_{n-1}$. But, once again, the number of restrictions is finite and we can place $a_{n-2}$ to produce the desired matrix representation of $M_{n}$.

\section{Unavoidability revisited}

Section 3 began with a discussion of unavoidable structures in graphs and developed the thread of an interesting interaction that occurs between graphs and matroids in the 2-connected case. There has been a similarly successful 
interaction in the 3-connected case, and this is described briefly in this section.

Corollary 3.3, which asserts that every large loopless 2-connected graph has a big cycle or a big bond, can be restated in terms of minors as follows. Recall that $C_{n}$ denotes an $n$-edge cycle, and let $C_{n}^{*}$ be the graph consisting of two vertices that are joined by $n$ edges.

Corollary 11.1 For each positive integer $n$, there is an integer $k(n)$ such that every 2-connected loopless graph with at least $k(n)$ edges has a minor isomorphic to $C_{n}$ or $C_{n}^{*}$.

The matroid generalization of this is an immediate consequence of Theorem 3.4. The cycle matroids of $C_{n}$ and $C_{n}^{*}$ are isomorphic to $U_{n-1, n}$ and $U_{1, n}$, respectively.

Corollary 11.2 For each positive integer $n$, there is an integer $k(n)$ such that every 2-connected matroid with at least $k(n)$ elements has a minor isomorphic to $U_{n-1, n}$ or $U_{1, n}$.

It is natural to ask what can be said about unavoidable minors in graphs and matroids when the connectivity is increased. One of the best-known and most important 3 -connected graphs is the $n$-spoked wheel $\mathcal{W}_{n}$. For $n \geq 3$, this graph is formed from an $n$-cycle, the rim, by adding an extra vertex and joining this to each vertex on the rim. The following result of Tutte [55] means that every simple 3-connected graph can be built from a wheel by adding edges or splitting vertices so that one maintains a 3-connected simple graph throughout.

Theorem 11.3 Let $n$ be an integer exceeding two. The following are equivalent for a simple 3-connected graph $G$ with $n+1$ vertices.

(i) For all edges e of $G$, neither $G \backslash$ e nor $G / e$ is both simple and 3-connected.

(ii) $G \cong \mathcal{W}_{n}$.

In view of this result, it is probably not surprising to see wheels occur among the set of unavoidable minors of 3-connected graphs. The following result was proved by Oporowski, Oxley, and Thomas [38].

Theorem 11.4 For each integer $n$ exceeding two, there is an integer $k(n)$ such that every 3-connected simple graph with at least $k(n)$ edges has a minor isomorphic to $\mathcal{W}_{n}$ or $K_{3, n}$.

Obtaining a matroid generalization of the last result proved to be quite difficult and followed a somewhat familiar pattern. The first generalization proved was to binary matroids [11]. We denote by $J_{n}$ and $\mathbf{1}$ the $n \times n$ and $1 \times n$ matrices of all ones. The vector matroid of the matrix $\left[I_{n}\left|J_{n}-I_{n}\right| \mathbf{1}\right]$ over $G F(2)$ is an $n$-spike with tip corresponding to the column $\mathbf{1}$. In general, $M\left[I_{n}\left|J_{n}-I_{n}\right| \mathbf{1}\right]$ is the unique binary spike of rank $n$. When $n=3$, this binary spike is the Fano matroid $F_{7}$. 
Theorem 11.5 For each integer $n$ exceeding two, there is an integer $k(n)$ such that every 3-connected binary matroid with at least $k(n)$ elements has a minor isomorphic to one of $M\left(\mathcal{W}_{n}\right), M\left(K_{3, n}\right), M^{*}\left(K_{3, n}\right)$, or $M\left[I_{n}\left|J_{n}-I_{n}\right| \mathbf{1}\right]$.

For 3-connected matroids in general, one would expect the list of unavoidable minors to grow and to include, in particular, the whirls, which we now define. In $M\left(\mathcal{W}_{n}\right)$, the rim is a circuit-hyperplane. By relaxing this, we obtain the rank-n whirl, $\mathcal{W}^{n}$. One of the fundamental results for 3-connected matroids, and another striking example of the successful generalization of a graph result to matroids, is Tutte's Wheels-and-Whirls Theorem [58], which is stated next.

Theorem 11.6 Let $n$ be an integer exceeding two. The following are equivalent for a 3-connected matroid $M$ of rank $n$.

(i) For all elements e of $M$, neither $M \backslash$ e nor $M / e$ is 3-connected.

(ii) $M$ is isomorphic to $M\left(\mathcal{W}_{n}\right)$ or $\mathcal{W}^{n}$.

The next result [12] extends Theorem 11.5 to arbitrary 3-connected matroids. Its proof, which required the development of some new tools, is outlined in $[40]$.

Theorem 11.7 For every integer $n$ exceeding two, there is an integer $k(n)$ such that every 3-connected matroid with at least $k(n)$ elements has a minor isomorphic to one of $M\left(\mathcal{W}_{n}\right), \mathcal{W}^{n}, M\left(K_{3, n}\right), M^{*}\left(K_{3, n}\right), U_{2, n+2}, U_{n, n+2}$, or an n-spike.

One may wonder whether the story stops with the 3-connected case. For matroids, the last result is as far as the theory has been developed. But, for graphs, Oporowski, Oxley, and Thomas [38] determined the families of unavoidable minors in the 4-connected case. Although their technique could possibly be extended to prove the corresponding result for 5-connected graphs, this has not been done. Moreover, a new technique will be needed to extend the result for $k$-connected graphs with $k \geq 6$. Let $n \geq 3$. We denote by $D_{n}$ the graph that is obtained from $\mathcal{W}_{n}$ by adding a new vertex and joining it to every vertex of the rim. The zig-zag circular ladder $Z_{n}$ is obtained from two vertex-disjoint cycles $u_{1} u_{2} \ldots u_{n}$ and $v_{1} v_{2} \ldots v_{n}$ by joining each $u_{i}$ to both $v_{i}$ and $v_{i+1}$, where $v_{n+1}=v_{1}$. The zig-zag Möbius ladder $V_{n}$ is obtained from a cycle $w_{1} w_{2} \ldots w_{2 n+1}$ by joining each $w_{i}$ to both $w_{i+n}$ and $w_{i+n+1}$, where all subscripts are interpreted modulo $2 n+1$. Hence $Z_{3}$ is the graph of the octahedron and $V_{3} \cong K_{5}$.

Theorem 11.8 For each integer $n$ exceeding three, there is an integer $k(n)$ such that every 4-connected simple graph with at least $k(n)$ edges has a minor isomorphic to one of $D_{n}, Z_{n}, V_{n}$, or $K_{4, n}$. 
Oporowski, Oxley, and Thomas also proved slightly stronger versions of Theorems 11.4 and 11.8 by establishing the corresponding results for topological minors.

We close this section with one further interesting development in this area that relates to graphs but not matroids. Corollary 11.1 can be strengthened as follows (see, for example, [38]). Notice that the magnitude condition is now one on vertices rather than edges, and the condition that the graph be loopless has been dropped. Similar modifications can be made to the other graph results appearing earlier in this section.

Theorem 11.9 For each positive integer $n$, there is an integer $k(n)$ such that every 2-connected graph with at least $k(n)$ vertices has a minor isomorphic to $C_{n}$ or $K_{2, n}$.

Instead of having two possible unavoidable classes, we could seek a result in which there was just a single such class. The following is an immediate consequence of Theorem 11.4. For a graph $H$, a graph $G$ has an $H$-minor if $G$ has a minor isomorphic to $H$.

Corollary 11.10 For each positive integer $n$, there is an integer $k(n)$ such that every 3-connected graph with at least $k(n)$ vertices has a $K_{1, n}$-minor.

Note that, while the goal of reducing to a single class of unavoidable minors has been achieved, we have lost the property that the members of that class maintain the connectivity of the starting graphs. The natural extension of the last result to 3-connected matroids would be that every sufficiently large 3-connected matroid $M$ has a big cocircuit. We know that this fails even when $M$ is regular since $M\left(K_{3, n}\right)$ has no circuits of size exceeding six so its dual has no cocircuits of size exceeding six.

Ding [9] has determined the connectedness needed to ensure that every sufficiently large graph has a big $K_{2, n}$-minor.

Theorem 11.11 For each positive integer $n$, there is an integer $k(n)$ such that every 5-connected graph with at least $k(n)$ vertices has a $K_{2, n}$-minor.

Ding has also conjectured the following natural extension of the last result, but he believes that he may not be the first to have conjectured this.

Conjecture 11.12 For each positive integer $n$, there is an integer $k(n)$ such that every 7-connected graph with at least $k(n)$ vertices has a $K_{3, n}$-minor.

Finally, Ding has significantly extended the last conjecture as follows.

Conjecture 11.13 There are functions $f(m)$ and $g(m, n)$ defined for all positive integers $m$ and $n$ such that the following hold:

(i) $f(m) \rightarrow \infty$ as $m \rightarrow \infty$;

(ii) $g(m, n) \rightarrow \infty$ as $n \rightarrow \infty$ for all fixed $m$; and

(iii) every $m$-connected graph with at least $n$ vertices has a $K_{f, g}$-minor. 


\section{Conclusion}

This paper highlights several broad areas in which interesting interactions occur between graphs and matroids. These relate to bounding the size of 2 connected matroids, finding removable circuits in 2- and 3-connected graphs and matroids, finding infinite antichains in matroids and graphs, and finding unavoidable classes of graph and matroid minors. Graph theorems that "can be expressed in terms of edges and circuits only" [56] are always going to suggest new matroid results. One of the aims of this paper has been to show that, by taking a matroid perspective on graphs, one can frequently produce new results not only for matroids but also for graphs.

\section{Acknowledgements}

The author thanks Bogdan Oporowski and Geoff Whittle for helpful discussions during the preparation of this paper. The author's work was partially supported by grants from the National Security Agency.

\section{References}

[1] R.E. Bixby, On Reid's characterization of the ternary matroids, J. Combin. Theory Ser. B 26 (1979), 174-204.

[2] J. Bonin, J. McNulty, and T. J. Reid, The matroid Ramsey number n(6,6), Combin. Probab. Comput. 8 (1999), 229-235.

[3] T. Brylawski, Constructions, in Theory of Matroids (eds. N. White), Encyclopedia of Math. and Its Applications, 26, Cambridge University Press, Cambridge (1986), pp. 127-223.

[4] T. Denley and T. J. Reid, On the number of elements in matroids with small circuits and small cocircuits, Combin. Probab. Comput. 8 (1999), $529-537$.

[5] J.S. Dharmatilake, Binary matroids of branch-width 3, Ph. D. thesis, Ohio State University, 1994.

[6] J.S. Dharmatilake, A min-max theorem using matroid separations, in $\mathrm{Ma}$ troid Theory (eds. J.E. Bonin, J.G. Oxley, B. Servatius), Contemporary Math., 197, Amer. Math. Soc., Providence (1996), pp. 333-342.

[7] R. Diestel, Graph Theory, Springer-Verlag, New York (1997).

[8] R. Diestel, T.R. Jensen, K.Y. Gorbunov, and C. Thomassen, Highly connected sets and the excluded grid theorem, J. Combin. Theory Ser. B 75 (1999), 61-73. 
[9] G. Ding, Graphs with no $K_{2, n}$ minor, Colloquium talk, Louisiana State University, 2000.

[10] G. Ding, B. Oporowski, and J. Oxley, On infinite antichains of matroids, J. Combin. Theory Ser. B 63 (1995), 21-40.

[11] G. Ding, B. Oporowski, J. Oxley, and D. Vertigan, Unavoidable minors of large 3-connected binary matroids, J. Combin. Theory Ser. B 66 (1996), 334-360.

[12] G. Ding, B. Oporowski, J. Oxley, and D. Vertigan, Unavoidable minors of large 3-connected matroids, J. Combin. Theory Ser. B 71 (1997), 244293.

[13] P. Erdős and G. Szekeres, A combinatorial problem in geometry, Compositio Math. 2 (1935), 463-470.

[14] P. Erdős and T. Gallai, On maximal paths and circuits of graphs, Acta Math. Acad. Sci. Hungar. 10 (1959), 337-356.

[15] H. Fleischner and B. Jackson, Removable cycles in planar graphs, J. London Math. Soc. (2) 31 (1985), 193-199.

[16] D.R. Fulkerson, Networks, frames, blocking systems, in Mathematics of the Decision Sciences, Part 1 Lectures in Applied Math., 11, Amer. Math. Soc., Providence (1968), pp. 303-334.

[17] J.F. Geelen, A.M.H. Gerards, and A. Kapoor, The excluded minors for GF(4)-representable matroids, J. Combin. Theory Ser. B 79 (2000), 247299.

[18] J.F. Geelen, A.M.H. Gerards, and G. Whittle, Branch width and wellquasi-ordering in matroids and graphs, Victoria University of Wellington, New Zealand, 2000.

[19] L.A. Goddyn and B. Jackson, Removable circuits in binary matroids, Combin. Probab. Comput. 6 (1999), 539-545.

[20] L.A. Goddyn, J. van den Heuvel, and S. McGuinness, Removable circuits in multigraphs, J. Combin. Theory Ser. B 71 (1997), 130-143.

[21] G. Higman, Ordering by divisibility in abstract algebras, Proc. London Math. Soc. 2 (1952), 326-336.

[22] F. Hurst and T.J. Reid, Some small circuit-cocircuit Ramsey numbers for matroids, Combin. Probab. Comput. 4 (1995), 67-80.

[23] F. Hurst and T.J. Reid, Ramsey numbers for cocircuits in matroids, Ars Combin. 45 (1997), 181-192. 
[24] B. Jackson, Removable cycles in 2-connected graphs of minimum degree at least four, J. London Math. Soc. (2) 21 (1980), 385-392.

[25] T. Johnson, N. Robertson, and P. Seymour, Grids in binary matroids of large branch width, Conference talk by T. Johnson, Oberwolfach, 1999.

[26] T. Johnson, N. Robertson, and P. Seymour, Grids in binary matroids, Conference talk by P. Seymour, Oberwolfach, 1999.

[27] J. Kruskal, Well-quasi-ordering, the tree theorem, and Vázsonyi's conjecture, Trans. Amer. Math. Soc. 95 (1960), 210-225.

[28] K. Kuratowski, Sur le problème des courbes gauches en topologie, Fund. Math. 15 (1930), 271-283.

[29] T. Lazarson, The representation problem for independence functions, $J$. London Math. Soc. 33 (1958), 21-25.

[30] A. Lehman, On the width-length inequality, Math. Programming 17 (1979), 403-417.

[31] M. Lemos and J. Oxley, On packing minors into connected matroids, Discrete Math. 189 (1998), 283-289.

[32] M. Lemos and J. Oxley, On removable circuits in graphs and matroids, J. Graph Theory 30 (1999), 51-66.

[33] M. Lemos and J. Oxley, On size, circumference and circuit removal in 3-connected matroids, Discrete Math. 220 (1999), 145-157.

[34] M. Lemos and J. Oxley, A sharp bound on the size of a connected matroid, Trans. Amer. Math. Soc., in press.

[35] W. Mader, Kreuzungfreie $a, b$-Wege in endlichen Graphe, Abh. Math. Sem. Univ. Hamburg 42 (1974), 187-204.

[36] S. McGuinness, Contractible bonds in bipartite graphs, University of Umeå, submitted, 1998.

[37] V. Neumann-Lara, E. Rivera-Campo, and J. Urrutia, A note on covering the edges of a graph with bonds, Discrete Math. 197/198 (1999), 633636.

[38] B. Oporowski, J. Oxley, and R. Thomas, Typical subgraphs of 3- and 4-connected graphs, J. Combin. Theory Ser. B 57 (1993), 239-257.

[39] J.G. Oxley, Matroid Theory, Oxford University Press, New York (1992). 
[40] J.G. Oxley, Unavoidable minors in graphs and matroids, in Graph Theory and Combinatorial Biology (eds. L. Lovász, A. Gyárfás, G. Katona, A. Recski, L. Székely), Bolyai Soc. Math. Stud., 7, János Bolyai Math. Soc., Budapest (1999), pp. 279-305.

[41] T.J. Reid, Ramsey numbers for matroids, Europ. J. Combin. 18 (1997), 589-585.

[42] N. Robertson and P.D. Seymour, Graph minors - a survey, in Surveys in Combinatorics 1985 (eds. I. Anderson), London Math. Soc. Lecture Notes, 103, Cambridge University Press, Cambridge (1985), pp. 153-171.

[43] N. Robertson and P.D. Seymour, Graph minors. IV. Tree-width and wellquasi-ordering, J. Combin. Theory Ser. B 48 (1990), 227-254.

[44] N. Robertson and P.D. Seymour, Graph minors. V. Excluding a planar graph, J. Combin. Theory Ser. B 41 (1986), 92-114.

[45] N. Robertson and P.D. Seymour, Graph minors. X. Obstructions to treedecomposition, J. Combin. Theory Ser. B 52 (1991), 153-190.

[46] N. Robertson and P.D. Seymour, Graph minors. XX. Wagner's conjecture, J. Combin. Theory Ser. B, in press.

[47] G.-C. Rota, Combinatorial theory, old and new, in Proc. Internat. Cong. Math. (Nice, 1970), Vol. 3, Gauthier-Villars, Paris (1971), pp. 229-233.

[48] P.D. Seymour, The matroids with the max-flow min-cut property, J. Combin. Theory Ser. B 23 (1977), 189-222.

[49] P.D. Seymour, Matroid representation over GF(3), J. Combin. Theory Ser. B 26 (1979), 159-173.

[50] P.D. Seymour, Decomposition of regular matroids, J. Combin. Theory Ser. B 28 (1980), 305-354.

[51] P.A. Sinclair, Strong snarks and the removal of edges from circuits in graphs, Ph. D. thesis, University of London, 1998.

[52] R. Thomas, A Menger-like property of tree-width: The finite case, $J$. Combin. Theory Ser. B 48 (1990), 67-76.

[53] C. Thomassen, A simpler proof of the excluded minor theorem for higher surfaces, J. Combin. Theory Ser. B 70 (1997), 306-311.

[54] W.T. Tutte, Matroids and graphs, Trans. Amer. Math. Soc. 90 (1959), $527-552$. 
[55] W.T. Tutte, A theory of 3-connected graphs, Nederl. Akad. Wetensch. Proc. Ser. A 64 (1961), 441-445.

[56] W.T. Tutte, Lectures on matroids, J. Res. Nat. Bur. Standards Sect. B 69B (1965), 1-47.

[57] W.T. Tutte, Menger's theorem for matroids, J. Res. Nat. Bur. Standards Sect. B 69B (1965), 49-53.

[58] W.T. Tutte, Connectivity in matroids, Canad. J. Math. 18 (1966), 13011324.

[59] Z. Tuza, On two intersecting set systems and $k$-continuous Boolean functions, Discrete Appl. Math. 16 (1987), 183-185.

[60] K. Wagner, Über eine Erweiterung eines Satzes von Kuratowski, Deut. Math. 2 (1937), 280-285.

[61] H. Whitney, On the abstract properties of linear dependence, Amer. J. Math. 57 (1935), 509-533.

[62] P.-L. Wu, An upper bound on the number of edges of a 2-connected graph, Combin. Probab. Comput. 6 (1997), 107-113.

[63] P.-L. Wu, Extremal graphs with prescribed circumference and cocircumference, Discrete Math. 223 (2000), 299-308.

Department of Mathematics Louisiana State University Baton Rouge, Louisiana 70803-4918 